\title{
Costly Monitoring, Dynamic Incentives, and Default*
}

\author{
Gaetano Antinolfi ${ }^{\dagger} \quad$ Francesco Carli ${ }^{\ddagger}$
}

September 19, 2011

\begin{abstract}
We study dynamic contracts between a lender and a borrower in the presence of costly state verification and hidden effort. The optimal contract minimizes social losses by mediating dynamic incentives and monitoring. Along the efficiency frontier, the threat of early termination is unavoidable for low levels of the borrower's promised utility; as the level increases, preventive monitoring is used to avoid future inefficient termination of the contractual relationship due to asymmetric information; for high level of promised utility, the threat of termination of the contractual relationship is no longer a useful tool to align dynamic incentives, preventive monitoring loses its role, and termination never occurs. Thus, the efficient contract optimizes the tradeoff between dymanic incentievs and static incentives. Following the interpretaion of the costly state verification literature, we can distinguish two levels of bankruptcy: one that leads to monitoring and the other that leads to liquidation.
\end{abstract}

\section{Introduction}

Financial contracts are typically written under conditions of asymmetric information and costly enforcement between lenders and borrowers. One of the

${ }^{*}$ We thank without implicating Francesca Carapella, Christian Ghiglino, Cyril Monnet, Daniel Sanches, Steve Spear, Steve Williamson, as well as seminar partcipants at the University of Essex, the University of Virginia, The Philly Fed, the Board of Governors, the 2011 Summer Meetings of the Econometric Society, and the reading group at Washington University.

${ }^{\dagger}$ Board of Governors of the Federal Reserve and Washington University in Saint Louis, gaetano@wustl.edu. Any views expressed are those of the authors and do not reflect the views of the Federal Reserve System.

${ }^{\ddagger}$ Washington University in Saint Louis, fcarli@wustl.edu. 
most important implications of these frictions is the possibility of strategic default. Two main assumptions that have been employed to model the possibility of strategic default are (i) that borrowers have limited commitment to repay, ${ }^{1}$ and (ii) that borrowers have private information over the realization of their projects and lenders must invest resources to observe it. ${ }^{2}$ In models where the lender-borrower relationship is affected by limited commitment on the part of the borrower, default is usually deterred through the threat of restricted access to credit in the future (for example, the termination of the contractual relationship). This mechanism relies on dynamic incentives and generates endogenous borrowing constraints which insure that default does not occur in equilibrium. This approach therefore exploits the value of future access to credit to enforce present contractual obligations. In models with costly state verification, on the other hand, monitoring is used by the lender to align incentives and to enforce repayment. Typically, albeit this is a relatively fragile result, the outcome of the minimization of the social loss incurred by costly monitoring is a standard debt contract, in which either the borrower repays a fixed amount or else defaults and the lender, upon auditing, becomes a residual claimant. Thus, the approach to the problem of strategic default in the prototypical costly state verification model is static, in the sense that current incentives are aligned through current punishment. Unlike models with limited commitment, models of costly state verification allow for default in equilibrium, where monitoring states are interpreted as default states. ${ }^{3}$ Moreover, default in these models is costly, because resources are expended to carry out the process of auditing and default.

In reality, both dynamic incentives and concurrent monitoring, are important in the contractual interaction between borrowers and lenders, and an important question is how they interact with one another or substitute one for the other. An easy way (conceptually, at least) to add dynamic incentives to a costly state verification environment is to make it dynamic. If the financing decision is repeated and current financing decisions can depend on history, dynamic incentives and monitoring can be combined together. Indeed, there is a relatively small literature that studies models of costly state verification in a dynamic setting. The assumption on preferences plays a crucial role. When principal and agent are risk neutral, Chang [5], Monnet and Quintin [10], and Webb [20] conclude that the optimal contract will fully

\footnotetext{
${ }^{1}$ An important reference is Kehoe and Levine [8].

${ }^{2}$ Some early papers are Townsend [18], Gale and Hellwig [6], and Williamson [21], [22].

${ }^{3}$ For a dynamic model with limited commitment where there is the possibility of default along the equilibrium path see Holpenhayn and Werning [7]. In their framework, an exogenous stochastic process that represents the evolution of an outside opportunity privately observed by the borrower induces a positive probability of default in every period.
} 
exploit the dynamic nature of the relationship: linearity of preferences allows agents to perfectly move consumption across time, monitoring consists of $\mathrm{s}$ social waste of resources, and the efficient way to minimize its costs is to link contracts together and make them depend on history. Dynamic incentives tend to crowd out monitoring, and monitoring, like default, can cease to be relevant in the limit in equilibrium. Thus, these results bring settings with costly state verification close to settings where there is limited commitment, in the sense that there is no equilibrium default and no interaction between monitoring and dynamic incentives.

The set of papers cited above all solve a present value problem where the optimal contract minimizes the intensity of monitoring, and hence its costs. When the borrower is risk averse, however, the entire distribution of repayments matters. The case of a risk averse borrower is considered by Smith and Wang [15] in a two-period setting and Wang [19] in an infinite horizon extension. They show that long-term contracts can provide some consumption smoothing across states without recurring to monitoring, but for this kind of insurance to be achieved promised future expected utilities must adjust to maintain incentive compatibility. The variation in promised future expected utilities results in lower intertemporal consumption smoothing, and the relevant trade-off for the lender is between the monitoring costs associated with complete insurance and the premium to the borrower, measured in expected utility, induced by the provision of incomplete insurance through dynamic incentives. With risk aversion, there is a limit in the provision of dynamic incentives because it is inefficient to set future promised utilities in future states too far apart. (The same principle makes termination of the contract inefficient.) In this class of models, Wang [19] transforms the dynamic problem into a sequence of static problems by considering deterministic monitoring probabilities and exponential utility, and in this case the set of monitoring states is time invariant: either a low income report is always verified or it is never verified. Differently, Popov [14] and Aiyagari and Alvarez [1], allow stochastic monitoring to obtain state dependent monitoring probabilities as a function, not necessarily monotonic, of the promised utility that the borrower is entitled to.

We abstract from the provision-of-insurance motive and we set up a model in which dynamic incentives and current monitoring interact to preserve future benefits from trade while deterring strategic default in the current period. The interesting aspect of this trade-off is that there are circumstances in which both monitoring and dynamic incentives are employed, and dynamic incentives alone cannot overcome informational asymmetries. Technically, we support the interaction between monitoring and dynamic incentives by assuming that, at the time of renewal of the loan, the borrower can affect 
the future probability of success of the investment project by choosing the level of effort devoted to the project. The decision over the level of effort is private information, and for this reason it generates an additional incentive compatibility constraint that increases the ability of the borrower to extract surplus from the relationship with the lender. Without this feature the model would resemble Monnet and Quintin [10], and monitoring and dynamic incentives could play only alternative roles, that is dynamic incentives would have solely the role of saving over monitoring costs and would always be preferred to monitoring, but would not otherwise interact in any interesting way. This kind of interaction between costly monitoring and dynamic incentives is the main result of our exercise. Intuitively, dynamic incentives tend to punish (or reward) indiscriminately, whereas monitoring allows a more precise form of punishment and reward. Monitoring provides the ability to distinguish whether a certain node in the history of the relationship between the principal and the agent was reached because of a sequence of bad shocks or because of shirking on the part of the agent. This piece of information is valuable to the principal, as it may prevent inefficient termination, but monitoring makes it costly to obtain. Thus, the efficient contract generates the optimal combination of dynamic incentives and monitoring.

One additional interpretation of the interaction between interim monitoring and dynamic incentives (in the form of the threat of termination) is that it leads to two levels or kinds of bankruptcy: one that leads to monitoring, and one that leads to liquidation (termination). Thus, one function performed by monitoring is to prevent project realizations and the information structure surrounding them from forcing inefficient liquidation. This is consistent with the literature on costly state verification models, in which monitoring states are interpreted as bankruptcy. In this model there is an additional form of bankruptcy: termination of the project. Thus, the model provides a way to distinguish between a mild form of bankruptcy, monitoring, and a stronger form of bankruptcy, liquidation or termination, as a function of the parameters in the problem.

Though we illustrate these ideas with relation to financial contracting, one could equally well think about a similar framework to study employment contracts. There are several examples in the literature, and Spear and Wang [16] has implications reminiscent of our results. In particular, Spear and Wang [16] explicitly considers, like we do, the possibility of termination. They conclude that there are two situations that lead to termination of the contract: a bad realization of the output of the worker (the CEO) or a very good realization. The intuition for these results is that after a low realization the CEO is too poor to be motivated effectively, whereas after a high realization it is essentially too expensive to provide the $\mathrm{CEO}$ with the 
appropriate incentives, and in both cases the firm is better off terminating the relationship and searching for a new CEO. In our model, the incentive compatibility constraint that arises from the borrower's decision over the effort level makes termination similar to the first case in Spear and Wang [16]: the borrower may become too poor to be motivated effectively after a low realization. Thus, even though the structure of our model is different from Spear and Wang [16], both exercises lead to results that underscore the importance of time and dynamics in contractual relationships.

In the next section we illustrate the model. In section 3 we provide the solution to the contracting problem and a discussion of the results.

\section{The model}

Time is discrete and consists of two periods, indexed by $t=1,2$. A principal (lender) and an agent (borrower) are both risk neutral and discount the future at the common rate $\delta$. Endowments and technologies are such that there are gains from trade in both periods.

The borrower has no wealth but has access to a stochastic technology that can be active in both periods: operating this technology in period $t$ requires a fixed investment $I_{t}=1$ at the beginning of the same time interval and returns the random realization $\theta_{t} \in\left\{\theta_{L}, \theta_{H}\right\}$ at the end of the period, where $\theta_{H}>\theta_{L}$. We assume that storage between periods is not possible. Hence, the borrower cannot invest in the second period without the help of the lender's funds. The random variables $\theta_{1}$ and $\theta_{2}$ are independent; we let the probability distribution of $\theta_{2}$ to be endogenously determined, while we assume the probability distribution of $\theta_{1}$ is exogenously given by $p=$ $\operatorname{Prob}\left(\theta_{1}=\theta_{H}\right)$. We assume that the borrower can influence the p.d.f. of $\theta_{2}$ in the second period through an unobservable effort choice $e \in\{0,1\}$. Define $p(e)=\operatorname{Prob}\left(\theta_{2}=\theta_{H} \mid e\right)$ and let $p(1)=p>p(0)$. Choosing an effort level $e$ has a cost $\psi(e)$ to the borrower; we normalize $\psi(1)=\psi>0=\psi(0)$. Finally, in both periods the project realization can be costlessly observed only by the borrower, whereas the lender has to incur a cost $\gamma$, expressed in utility terms, to observe it.

The lender has positive wealth at the beginning of each period and has access to a safe technology that in return for a unit investment at the beginning of the period yields $(1+i)$ units of the consumption good at the end of the same period. For simplicity, we normalize $i=0$.

We assume the lender is committed, at the beginning of the first period, to deliver the borrower an expected utility $w$ (later we will carefully specify its possible values); we assume that the lender can commit over future actions, 


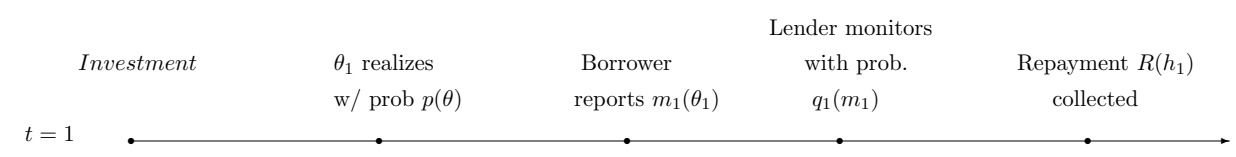

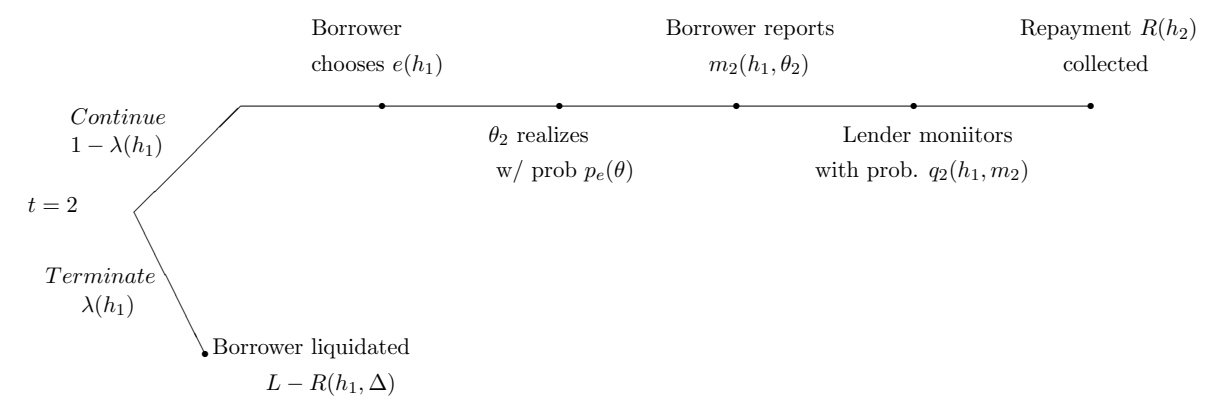

Figure 1: Time line of events

but the borrower cannot. The timing of events are described in Figure 1: it is always optimal to operate the borrower's technology in the first period, which yields the random return $\theta_{1}$. After observing it, the borrower reports the message $m_{1}$ to the lender who monitors the true income realization with probability $q_{1}$. Repayments are collected, both consume and the game moves to $t=2$. At the beginning of the second period, the lender can terminate the relationship or continue it. If the relationship doesn't terminate, the borrower optimally chooses the effort level $e \in\{0,1\}$ and, as in the first period, after the project outcome is realized, the borrower reports the message $m_{2}$, the lender monitors the project realization with probability $q_{2}$, repayments are collected and both consume. If the relationship is terminated the borrower receives the termination payment $L$ and both consume.

Given a consumption stream $\left(c_{1}, c_{2}\right)$ and a second period effort level $e$, the borrower's lifetime utility is $u^{B}\left(c_{1}, c_{2} ; e\right)=c_{1}+\delta\left(c_{2}-\psi e\right)$. The lender's lifetime utility is instead defined over consumption streams $\left(c_{1}, c_{2}\right)$ and monitoring effort $\left(\gamma_{1}, \gamma_{2}\right): u^{L}\left(c_{1}, c_{2} ; \gamma_{1}, \gamma_{2}\right)=\left(c_{1}-\gamma_{1}\right)+\delta\left(c_{2}-\gamma_{2}\right)$.

We introduce the following assumption to make the solution of the problem a non-trivial one:

Assumption 1. $p(0) \theta_{H}+\left((1-p(0)) \theta_{L}<1<p \theta_{H}+(1-p) \theta_{L}-\psi\right.$

On the one hand, this assumption is necessary to have a positive expected surplus from trade: the second inequality puts an upper bound on the effort 
$\operatorname{cost} \psi$. If this upper bound wasn't satisfied there would always be an aggregate loss from operating the borrower's technology, and it would never be optimal for the lender to finance the borrower's project in the second period. On the other hand, this assumption makes the effort choice relevant to the lender's termination decision, because the aggregate surplus from trade vanishes if the borrower chooses a level of effort equal to zero. If this inequality were not satisfied, it would always be optimal for the lender to finance the borrower's project (as long as monitoring costs are reasonably small).

\section{Optimal contract}

Let $h_{1}$ and $h_{2}$ be respectively the first and second period public information: $h_{1}$ is a pair $\left(m_{1}, a_{1}\right)$ where $m_{1} \in\left\{\theta_{L}, \theta_{H}\right\}$ is the borrower's reported income (we consider contracts that use direct revelation mechanism) and $a_{1} \in \mathcal{A}=$ $\left\{\emptyset, \theta_{L}, \theta_{H}\right\}$ is the information about the true project realization $\theta_{1}$ acquired by the lender ( $a_{1}=\emptyset$ means no verification is carried out). Time-two public information $h_{2}$ is defined in the same way if the relationship is not terminated: $h_{2}=\left(m_{2}, a_{2}\right)$. We use the notation $h_{2}=\Delta$ to represent the state in which the relationship is terminated and the borrower's technology is not operated in the second period.

Define $h^{1}=h_{1}$ and $h^{2}=\left(h_{1}, h_{2}\right)$ to be respectively time- 1 and time-2 public histories and let $\mathcal{H}_{t}$, for $t=1,2$, be the collection of all possible public histories $h^{t}$ at time $t$. A contract is a collection $\sigma=\left\{q_{t}\left(h^{t-1}, m_{t}\right), R_{t}\left(h^{t}\right)\right.$, $\left.\lambda\left(h^{1}\right), e\left(h^{1}\right), L\left(h^{1}, \Delta\right) ; t=1,2 ; \forall h^{1}\right\}$ where $q_{t}: \mathcal{H}_{t-1} \times \Theta \rightarrow[0,1]$ maps past histories and current announcements into monitoring probabilities, $R_{t}: \mathcal{H}_{t} \rightarrow$ $\Re$ maps histories into repayments if the borrower operates the investment technology $\left(h_{2} \neq \Delta\right), \lambda: \mathcal{H}_{1} \rightarrow[0,1]$ is the probability of termination, $e$ : $\mathcal{H}_{1} \rightarrow\{0,1\}$ is the second period suggested effort level, and $L: \mathcal{H}_{1} \times\{\Delta\} \rightarrow \Re$ is the payment the borrower receives in the event of termination.

We assume that repayments cannot be negative and cannot exceed the project's realization (the announced realization if the true one is not monitored). We additionally assume that the borrower is protected by limited liability if the project is terminated:

$$
L\left(h^{1}, \Delta\right) \geq 0 \quad 0 \leq R_{t}\left(h^{t}\right) \leq \begin{cases}\theta_{i} & \text { if } h_{t}=\left(\theta_{i}, \phi\right) \\ \theta_{j} & \text { if } h_{t}=\left(\theta_{i}, \theta_{j}\right) .\end{cases}
$$

We consider contracts that are implemented by perfect public equilibria (in the second period the borrower could potentially condition his strategies on $z_{1}=\left(\theta_{1}, m_{1}, a_{1}\right)$, the first period private information). A strategy profile for the borrower consists in reporting functions $m_{1}:\left\{\theta_{L}, \theta_{H}\right\} \rightarrow\left\{\theta_{L}, \theta_{H}\right\}$, 
$m_{2}: h^{1} \times\left\{\theta_{L}, \theta_{H}\right\} \rightarrow\left\{\theta_{L}, \theta_{H}\right\}$ and an effort choice $e: h^{1} \rightarrow\{0,1\}$. Define $\Omega=\left\{m_{1}\left(\theta_{1}\right), m_{2}\left(h^{1}, \theta_{2}\right) ; e\left(h^{1}\right)\right\}$ to be the collection of all possible reportingeffort strategy profiles.

Given a sequence of realizations $\left(\theta_{1}, \theta_{2}\right)$, an effort choice function $e$ and a contract $\sigma$, we say a reporting strategy $\left(m_{1}, m_{2}\right) \in\left\{\theta_{L}, \theta_{H}\right\}^{2}$ is feasible for $\left(\theta_{1}, \theta_{2}\right)$ if $R_{1}\left(h^{1}\right) \leq \theta_{1}$ and $R_{2}\left(h^{2}\right) \leq \theta_{2}$, where $h^{1}=\left(m_{1}, a_{1}\right)$ is any time-one public history reached with positive probability following the message $m_{1}$ (and realization $\theta_{1}$ ); for any of these histories $h^{1}$ we require $h^{2}=\left(h^{1}, m_{2}, a_{2}\right)$ to be reached with positive probability after a message $m_{2}$ has been sent (effort $e$ has been exerted and $\theta_{2}$ has realized). Let $\mathcal{F}_{\left(\theta_{1}, \theta_{2}\right)} \subseteq\left\{\theta_{L}, \theta_{H}\right\}^{2}$ be the collection of such feasible reports. The limited liability constraint above makes any report feasible if the true project realization is verified. Therefore, the set of feasible reporting is $\mathcal{F}_{\left(\theta_{1}, \theta_{2}\right)}=\left\{\left(m_{1}, m_{2}\right): R_{1}\left(m_{1}, \emptyset\right) \leq \theta_{1}\right.$, if $q_{1}\left(m_{1}\right)<1$ and given $h^{1}=\left(m_{1}, a_{1}\right)$ for any possible $a_{1}, R_{2}\left(h^{1},\left(m_{2}, \emptyset\right)\right) \leq \theta_{2}$, if $\left.q_{2}\left(h^{1}, m_{2}\right)<1\right\}$. Define $\Omega_{\mathcal{F}} \subseteq \Omega$ to be the collection of reporting-effort strategies with the reporting plan $\left(m_{1}, m_{2}\right) \in \mathcal{F}_{\left(\theta_{1}, \theta_{2}\right)}$ being feasible for all possible $\left(\theta_{1}, \theta_{2}\right) \in\left\{\theta_{L}, \theta_{H}\right\}^{2}$.

For a feasible reporting-effort plan $\left(m_{1}, m_{2}, e\right)$, and a feasible contract $\sigma$

$$
V\left(m, e, \sigma ; h^{1}\right)=\lambda\left(h^{1}\right) L\left(h^{1}\right)+\left(1-\lambda\left(h^{1}\right)\right) E_{z_{2}}\left[\theta_{2}-R_{2}\left(h^{1}, h_{2}\right) \mid h^{1}\right]
$$

and

$$
\begin{array}{r}
V\left(m, e, \sigma ; h^{0}\right)=E_{\left(z_{1}, z_{2}\right)}\left[\left(\theta_{1}-R_{1}\left(h^{1}\right)\right)+\delta\left\{\lambda\left(h^{1}\right) L\left(h^{1}\right)\right.\right. \\
\left.\left.+\left[1-\lambda\left(h^{1}\right)\right]\left[\theta_{2}-R_{2}\left(h^{1}, h^{2}\right)\right]\right\}\right]
\end{array}
$$

are respectively the borrower's payoffs for the game starting after history $h^{1}$ and for the whole game, where the expectations are computed over private histories $\left(z_{1}, z_{2}\right)$, for $z_{1}=\left(\theta_{1}, m_{1}, a_{1}\right)$ defined above and $z_{2}=\left(e, \theta_{2}, m_{2}, a\right)$ in the case the relationship continues and $z_{2}=\Delta$ if not.

A contract $\sigma$ is incentive compatible if at any history truth-telling $m_{t}^{*}\left(h^{t-1}\right.$, $\left.\theta_{t}\right)=\theta_{t}$ and the effort level suggested by the contract $e^{*}\left(h^{1}\right)$ are the best feasible strategies for the borrower:

$$
V\left(m^{*}, e^{*}, \sigma ; h^{t-1}\right) \geq V\left(\widehat{m}, \widehat{e}, \sigma ; h^{t-1}\right) \forall(\widehat{m}, \widehat{e}) \in \Omega_{\mathcal{F}}, \forall h^{t-1}
$$


The optimal contract maximizes the lender's expected revenues,

$$
\begin{aligned}
\max _{\sigma} & E_{\left(h_{1}, h_{2}\right)}\left[\left(R_{1}\left(h_{1}\right)-\gamma_{1}\left(h^{1}\right)\right)\right. \\
& \left.+\delta\left\{\left[1-\lambda\left(h^{1}\right)\right]\left(R_{2}\left(h^{2}\right)-\gamma_{2}\left(h^{2}\right)\right)-\lambda\left(h^{1}\right)\left[1-L\left(h^{1}, \Delta\right)\right]\right\}\right]
\end{aligned}
$$

subject to incentive compatibility (2), the limited liability constraints (1) and contingent on delivering the initial promised expected utility $w_{0}, V\left(m^{*}, e^{*}, \sigma ; h^{0}\right)=$ $w_{0} ; \gamma_{t}\left(h^{t}\right)$ is the lender time $t$ monitoring effort given history $h^{t}$ and equals $\gamma$ if the project realization is observed and zero if not. It is easy to show that the problem can be more conveniently solved recursively using the borrower's promised expected utility as a state variable.

\subsection{The second period contract}

At the beginning of the second period, the borrower is entitled to a promised expected utility $w_{2}$. Depending on its value, the lender can choose to terminate the relationship, pay the borrower the amount $w_{2}$ and invest whatever left in the safe technology, or continue the relationship and finance the borrower's project, incurring the fixed cost $I_{2}=1$. We allow for the possibility of randomizing over the two strategies, therefore the contract takes the form of a triplet $\left(\lambda, w^{C}, w^{T}\right)$ where $\lambda\left(w_{2}\right)$ is the probability of termination, $w^{C}\left(w_{2}\right)$ and $w^{T}\left(w_{2}\right)$ are respectively the borrower's promised utility if the lender finances the project or if the relationship is terminated. The lender's value function in the second period, $\Pi_{2}\left(w_{2}\right)$, is given by

$$
\begin{gathered}
\Pi_{2}\left(w_{2}\right)=\max _{\lambda, w^{C}, w^{T}} \lambda \Pi_{2}^{T}\left(w^{T}\right)+(1-\lambda) \Pi_{2}^{C}\left(w^{C}\right) \\
\lambda w^{T}+(1-\lambda) w^{C}=w_{2} \\
0 \leq w^{T} \quad 0 \leq w^{C} \leq \bar{w}
\end{gathered}
$$

Equation (4) is a standard promise-keeping constraint. Equation (5) is a limited liability constraint that limits the punishment the borrower can possibly receive in the case of termination, whereas in the case the partnership continues it simply states that repayments have to be a positive fraction of the project's realization. 
The lender will compare the expected return from investing one unit of his wealth to operate the borrower's technology with the outside opportunity of investing it in the safe storage technology, conditional on honoring in both cases the promise made to deliver the borrower the expected utility $w_{2}$. Therefore, $\Pi_{2}^{T}\left(w^{T}\right)=1-w^{T}$ is the lender expected revenue if the partnership is terminated, whereas $\Pi_{2}^{C}\left(w^{C}\right)$ comes from the inner maximization in case the partnership continues. In this case, the contract will specify a suggested effort level $e: \Re_{+} \rightarrow\{0,1\}$, a monitoring probability $q: \Re_{+} \times \Theta \rightarrow[0,1]$, and the repayment functions $r: \Re_{+} \times \Theta \rightarrow \Re_{+}$when the project outcome is not monitored, and $R: \Re_{+} \times \Theta \times \Theta \rightarrow \Re_{+}$if the outcome is monitored:

$$
\begin{gathered}
\Pi_{2}^{C}\left(w^{C}\right)=\max _{e, q_{i}, R_{i, j}, r_{i}} \sum_{i=H, L} p_{i}(e)\left[q_{i} R_{i, i}+\left(1-q_{i}\right) r_{i}-\gamma q_{i}\right] \\
\text { s.t. } \sum_{i=L, H} p_{i}(e)\left\{\theta_{i}-\left[q_{i} R_{i, i}+\left(1-q_{i}\right) r_{i}\right]\right\}-\psi(e)=w^{C} \\
\theta_{i}-\left(q_{i} R_{i, i}+\left(1-q_{i}\right) r_{i}\right) \geq \theta_{i}-\left(q_{j} R_{i, j}+\left(1-q_{j}\right) r_{j}\right) \text { if } r_{j} \leq \theta_{i} \\
\qquad \underset{\widehat{e} \in\{0,1\}}{\operatorname{argmax}}\left\{\sum_{i=L, H} p_{i}(\widehat{e})\left\{\theta_{i}-\left[q_{i} R_{i, i}+\left(1-q_{i}\right) r_{i}\right]\right\}-\psi(\widehat{e})\right\} \\
0 \leq R_{i, j}, r_{i} \leq \theta_{i} \quad 0 \leq q_{i} \leq 1
\end{gathered}
$$

Equation (7) is the promise-keeping constraint of the inner maximization, where $R_{i, j}$ is the borrower's repayment when the lender inspects the project realization and discovers this is $\theta_{i}$ after the borrower announced $\theta_{j}$, whereas $r_{i}$ is the repayment when the project realization is not monitored and the borrower announced it to be $\theta_{i}$. Constraint (9) is the incentive compatibility constraint for the recommended effort level, (8) is the incentive compatibility that guarantees that the borrower will truthfully report the project realization (for the cases when misreporting is feasible), and (10) makes the probabilities of monitoring meaningful and it additionally consists of a twosided limited liability constraint that restricts repayment to be a positive fraction of the project realization.

The inner maximization solves a static costly state verification problem with the additional constraint (9) that distinguishes repayments that are compatible with a positive effort level $e=1$ from repayments that are not compatible with it. As it will be described later, this constraint generates a partition 
in the space of feasible promised utilities $w_{2}$ that are compatible with effort $e=1$ and with effort $e=0$.

It is always optimal to make $R_{i j}=\theta_{i}$, for $i \neq j$, because this relaxes the incentive compatibility constraint (8) and the variable $R_{i j}$ does not enter in the objective or any other constraint. Moreover, the fact that there are only two possible realizations simplifies the truth-telling constraint (8).

Lemma 1. For $i \neq j$ it is optimal to set $R_{i, j}=\theta_{i}$. No monitoring ever happens if $\theta_{H}$ is announced: $q_{H}=0$. The incentive compatibility (8) binds for $\theta_{i}=\theta_{H}$ and can be ignored for $\theta_{i}=\theta_{L}$.

Because of Lemma 1, the relevant constraints for the contract, contingent on continuation, are the promise keeping constraint (7), that can be rewritten as

$p(e) \theta_{H}+(1-p(e)) \theta_{L}-\left[\left(1-q_{L}\right) r_{L}+q_{L}\left(p(e) \theta_{H}+(1-p(e)) R_{L L}\right)\right]-\psi(e)=w$,

and the incentive compatibility constraint (9) that, for $e=1$, becomes

$$
\left(\theta_{H}-r_{H}\right)-\left\{q_{L}\left(\theta_{L}-R_{L L}\right)+\left(1-q_{L}\right)\left(\theta_{L}-r_{L}\right)\right\} \geq \frac{\psi}{p-p(0)}
$$

Define $\bar{\theta}_{e}=p(e) \theta_{H}+(1-p(e)) \theta_{L}$ to be the project expected return conditional on the effort level $e \in\{0,1\}$, and let $\widehat{w}=p\left(\theta_{H}-\theta_{L}\right)-\psi$ and $\widetilde{w}=\frac{p(0)}{p-p(0)} \psi$ to be two thresholds on the promised utility $w_{2}$. Notice that $\widehat{w}>\widetilde{w}$ by Assumption 1 .

Lemma 2. The continuation contract is compatible with a positive effort level $e=1$ if and only if $w \geq \widetilde{w}$. The probability of monitoring is $q_{L}=0$ if $w \geq \widehat{w}$ and $q_{L}=1-\frac{w+\psi(e)}{p(e)\left(\theta_{H}-\theta_{L}\right)}$ if $w<\widehat{w}$.

Proof. The first thing to notice is that we can set $R_{L L}=\theta_{L}$, because in this way the constraint (9) is relaxed, and if $q_{L}>0$ this saves on monitoring costs. Second, recall that $\widehat{w}=p\left(\theta_{H}-\theta_{L}\right)-\psi$ : when $w \geq \widehat{w}$ the promise keeping constraint implies

$$
\left(1-q_{L}\right) r_{L}+q_{L}\left[p(e) \theta_{H}+(1-p(e)) R_{L L}\right] \leq \theta_{L},
$$

and looking at the objective (6) we conclude that setting $q_{L}=0$ and $r_{L}=$ $\bar{\theta}_{1}-w-\psi$ saves on monitoring costs. Moreover, by Assumption 1 such a contract is compatible with $e=1$, therefore it is the optimal one.

When $w<\widehat{w}$ choosing $q_{L}=0$ is not possible because it would violate the 
limited liability constraint for $r_{L}$. After replacing $r_{L}=\theta_{L}$, we obtain from the promise keeping constraint

$$
q_{L}(w)=1-\frac{w+\psi(e)}{p(e)\left(\theta_{H}-\theta_{L}\right)} .
$$

The only thing left to determine is whether the monitoring and the repayment scheme is compatible with a positive effort level $e=1$. Looking at the incentive compatibility constraint it follows that this is not the case for $w<$ $\widetilde{w}=\frac{p(0)}{p-p(0)} \psi$.

As a corollary, the function $\Pi^{C}(w)=\bar{\theta}_{1}-w$ if $w \in[\widehat{w}, \bar{w}], \Pi^{C}(w)=$ $\bar{\theta}_{1}-w-\gamma(1-p)\left(1-\frac{w+\psi}{p\left(\theta_{H}-\theta_{L}\right)}\right)$ if $w \in[\widetilde{w}, \widehat{w})$, and $\Pi^{C}(w)=\bar{\theta}_{0}-w-\gamma(1-$ $p(0))\left(1-\frac{w}{p(0)\left(\theta_{H}-\theta_{L}\right)}\right)$ if $w \in[0, \widetilde{w})$. Using this functional form, it is now possible to go back to problem (3) and solve for the optimal probability of termination and the promised expected utilities $w^{C}$ and $w^{T}$ :

Proposition 1. There is a threshold $w^{*}(\gamma)$ on the promised utility $w_{2}$ such that the relationship is never terminated if $w_{2} \geq w^{*}$, whereas it is terminated with some probability if $w_{2}<w^{*}$. The borrower is entitled to zero utility if the contract terminates while he receives a promised expected utility $w^{C} \geq w^{*}$ if the contract continues (with equality if $\lambda\left(w_{2}\right)>0$ ).

Proof. Looking at equation (3) and (4), observe first that it must hold that $w^{C} \geq \widetilde{w}$. Indeed, if this was not the case, we could define a new contract with $\lambda^{\prime}=1$ and $w^{T^{\prime}}=\lambda w^{T}+(1-\lambda) w^{C}$ and the expected revenues $\Pi^{\prime}\left(w_{2}\right)$ of the new contract would be

$$
\begin{aligned}
\Pi^{\prime}\left(w_{2}\right) & =1-w^{T^{\prime}}=1-\lambda w^{T}-(1-\lambda) w^{C}=\lambda\left(1-w^{T}\right)+(1-\lambda)\left(1-w^{C}\right) \\
& >\lambda\left(1-w^{T}\right)+(1-\lambda) \Pi_{2}^{C}\left(w^{C}\right)=\Pi\left(w_{2}\right)
\end{aligned}
$$

Second, $\lambda=1$ if and only if $w_{2}=0$. If $w_{2}=0, \lambda=1$ follows from the previous point, the promise keeping constraint (4) and the limited liability constraint (5). To prove the only if part suppose by contradiction that $w_{2}>0$ and $\lambda=1$, and consider a new contract with $w^{C^{\prime}}=\max \left\{w_{2}, \widehat{w}\right\}, \lambda^{\prime}=\max \left\{0, \frac{\widehat{w}-w_{2}}{\widehat{w}}\right\}$ and $w^{T^{\prime}}=0$. Trivially, if $w_{2}>\widehat{w}, \Pi^{\prime}\left(w_{2}\right)=\Pi^{C}\left(w_{2}\right)>1-w_{2}=\Pi\left(w_{2}\right)$. If $w_{2} \leq \widehat{w}$ instead

$$
\begin{aligned}
\Pi^{\prime}\left(w_{2}\right) & =1+\frac{w_{2}}{\widehat{w}}\left(\Pi^{C}(\widehat{w})-1\right)=1-w_{2}+w_{2}\left(\frac{\Pi^{C}(\widehat{w})-1}{\widehat{w}}+1\right) \\
& =\left(1-w_{2}\right)+w_{2}\left(\frac{\Pi^{C}(\widehat{w})+\widehat{w}-1}{\widehat{w}}\right)>\left(1-w_{2}\right)=\Pi\left(w_{2}\right)
\end{aligned}
$$


Third, if $\lambda>0$ it must hold that $w^{T}<w^{C}$.

Indeed, suppose that $w^{T} \geq w^{C}$ and $\lambda>0$. We already know that $w^{C} \geq \widetilde{w}$, therefore two scenarios are possible: $w^{C}<\widehat{w}$ or $w^{C} \geq \widehat{w}$. In the first case, define $w^{T^{\prime}}=w^{T}-\epsilon$ and $w^{C^{\prime}}=w^{C}-\epsilon \frac{\lambda}{1-\lambda}$. Then the expected revenues would be $\Pi^{\prime}\left(w_{2}\right)=\Pi\left(w_{2}\right)+\epsilon \lambda(1+\phi)>\Pi\left(w_{2}\right)$, where $\phi>-1$ is the slope of $\Pi_{2}^{C}(\cdot)$. In the second case define $\lambda^{\prime}=0$ and $w^{C^{\prime}}=\lambda w^{T}+(1-\lambda) w^{C}$. The expected revenues associated with the new contract are $\Pi^{\prime}\left(w_{2}\right)=\Pi\left(w_{2}\right)+$ $\lambda\left(\Pi^{C}\left(w^{C}\right)+w^{C}-1\right)>\Pi\left(w_{2}\right)$.

Last observation, if $\lambda>0$ it must be $w^{T}=0$. If not, and $w^{C}<w_{2}$, define a new contract with $w^{T^{\prime}}=0$ and $w^{c^{\prime}}=w^{C}+\frac{\lambda}{1-\lambda} w^{T}$. Then,

$$
\begin{aligned}
\Pi^{\prime}\left(w_{2}\right) & =(1-\lambda) \Pi^{C}\left(w^{C^{\prime}}\right)+\lambda=(1-\lambda) \Pi^{C}\left(w^{C}+\frac{\lambda}{1-\lambda} w^{T}\right)+\lambda \\
& >(1-\lambda) \Pi^{C}\left(w^{C}\right)-\lambda w^{T}+\lambda=(1-\lambda) \Pi^{C}\left(w^{C}\right)+\lambda\left(1-w^{T}\right)=\Pi\left(w_{2}\right)
\end{aligned}
$$

where the inequality follows from $w^{C}<w_{2}$. If instead $w^{C} \geq w_{2}$ consider the new contract with $w^{T^{\prime}}=0$ and $\lambda^{\prime}=\lambda\left(1-\frac{w^{T}}{w^{C}}\right)<\lambda$. The expected revenues are

$$
\begin{aligned}
\Pi^{\prime}\left(w_{2}\right) & =(1-\lambda) \Pi^{C}\left(w^{C^{\prime}}\right)+\lambda^{\prime}=\lambda\left(1-\frac{w^{T}}{w^{C}}\right)+\left(1-\lambda\left(1-\frac{w^{T}}{w^{C}}\right)\right) \Pi^{C}\left(w^{C}\right) \\
& \left.\geq \Pi\left(w_{2}\right)+\frac{w^{T}}{w^{C}} \lambda\left(\Pi^{C}\left(w^{C}\right)+w^{C}\right)-1\right)>\Pi\left(w_{2}\right)
\end{aligned}
$$

Replacing $w^{T}=0$ in the promise keeping constraint (4) we get

$$
(1-\lambda) w^{C}=w_{2}
$$

which implies that $w^{C} \geq w_{2}$, with strict inequality if $\lambda>0$. Solving for $\lambda$ we get $\lambda=\frac{w^{C}-w_{2}}{w^{C}}$ and replacing it in the objective (3) we have

$$
\begin{gathered}
\Pi_{2}\left(w_{2}\right)=\max _{w^{C}} 1+\frac{w_{2}}{w^{C}}\left(\Pi^{C}\left(w^{C}\right)-1\right) \\
\text { s.t. } \quad \bar{w} \geq w^{C} \geq w_{2} .
\end{gathered}
$$

This problem can be easily solved, separately, for $w_{2} \geq \widehat{w}$ and for $0 \leq w_{2}<\widehat{w}$. If $w_{2} \geq \widehat{w}$, the objective is differentiable over the entire interval and its derivative is

$$
\frac{w_{2}}{\left(w^{C}\right)^{2}}\left[1-\left(\Pi^{C}\left(w^{C}\right)+w^{C}\right)\right]
$$

where the term in parenthesis is always strictly negative and the only solution is $w^{C}=w_{2}$ (therefore $\left.\lambda=0\right)$. 
If $0<w_{2}<\widehat{w}$, we can assume without loss of generality that $\widehat{w} \leq w^{C} \leq \widehat{w}$ and the objective is differentiable over such an interval, and its first derivative is

$$
\frac{w_{2}}{\left(w^{C}\right)^{2}}\left[1-\left(\Pi^{C}\left(w^{C}\right)-\phi_{C} w^{C}\right)\right]=\frac{w_{2}}{\left(w^{C}\right)^{2}}\left[1-A_{C}\right],
$$

where $\phi_{C}$ and $A_{C}$ are respectively the slope and the intercept of $\Pi^{C}(\cdot)$ for $w^{C} \in[\widetilde{w}, \widehat{w}]$. Depending on the sign of the term in parenthesis, either $w^{C}=\widehat{w}$ for all $w_{2}<\widehat{w}$ (if $1>A_{C}$ ) or $w^{C}=w_{2}$ for $\widetilde{w} \leq w_{2} \leq \widehat{w}$ and $w^{C}=\widetilde{w}$ for $w_{2}<\widetilde{w}\left(\right.$ if $\left.A_{C}>1\right)$.

Since $A_{C}(\gamma)$ is a monotone decreasing function of the monitoring costs $\gamma$ not bounded below and $A_{C}(0)>1$, there is a threshold $\infty>\widehat{\gamma}>0$ such that the solution is of the first type described above for $\gamma>\widehat{\gamma}$ while it is of the second type for $\gamma \leq \widehat{\gamma}$, which concludes the proof.

Notice that the optimal termination threshold $w^{*}$ depends exclusively on the difference $\left(A_{C}-1\right)$, implying that the the slope of the value function $\Pi_{2}$ is weakly decreasing: for $w_{2} \geq w_{2}^{\prime}, \phi\left(w_{2}\right) \leq \phi\left(w_{2}^{\prime}\right) .{ }^{4}$ This means that the value function $\Pi_{2}(\cdot)$ is piecewise linear, but concave overall (Figure 2).

Termination is always part of the optimal contract when the promised utility $w_{2}$ is low enough, because the limited liability constraint restricts the set of feasible repayments and therefore the ability of the lender to shape the continuation contract to induce a positive level of effort. This means that, when the contract must make the worker sufficiently poor in the second period, termination is a necessary punishment device, because the lender won't find attractive anymore to finance the borrower's project.

It is also possible, but not very interesting, that a positive probability of termination is optimal for an additional set of promised utilities. This type of termination emerges when the costs of verification are extremely high and a considerable part of the joint surplus that would be created by operating the borrower's project would be consumed by monitoring. It follows the lender is better off randomizing over termination, avoiding in this way any verification effort.

Even when satisfying the weak concavity property, the value function $\Pi_{2}$ can have very different shapes. Figure 2 depicts the two extreme cases. In the first situation the cost of monitoring $\gamma$ and the effort costs $\psi$ are both low enough that termination introduces a non-monotonicity in the value function. The second scenario corresponds to the high monitoring costs case and the value function is monotone decreasing. In general few other cases are possible, with the costs of monitoring $\gamma$ determining the slope of the value function in

\footnotetext{
${ }^{4}$ Obviously the slope is not defined at the kinks.
} 
the intermediate region (hence the slope in the first region) while the effort cost $\psi$ and the probability $p(0)$ determine the distance between $\widehat{w}$ and $\widetilde{w}$.

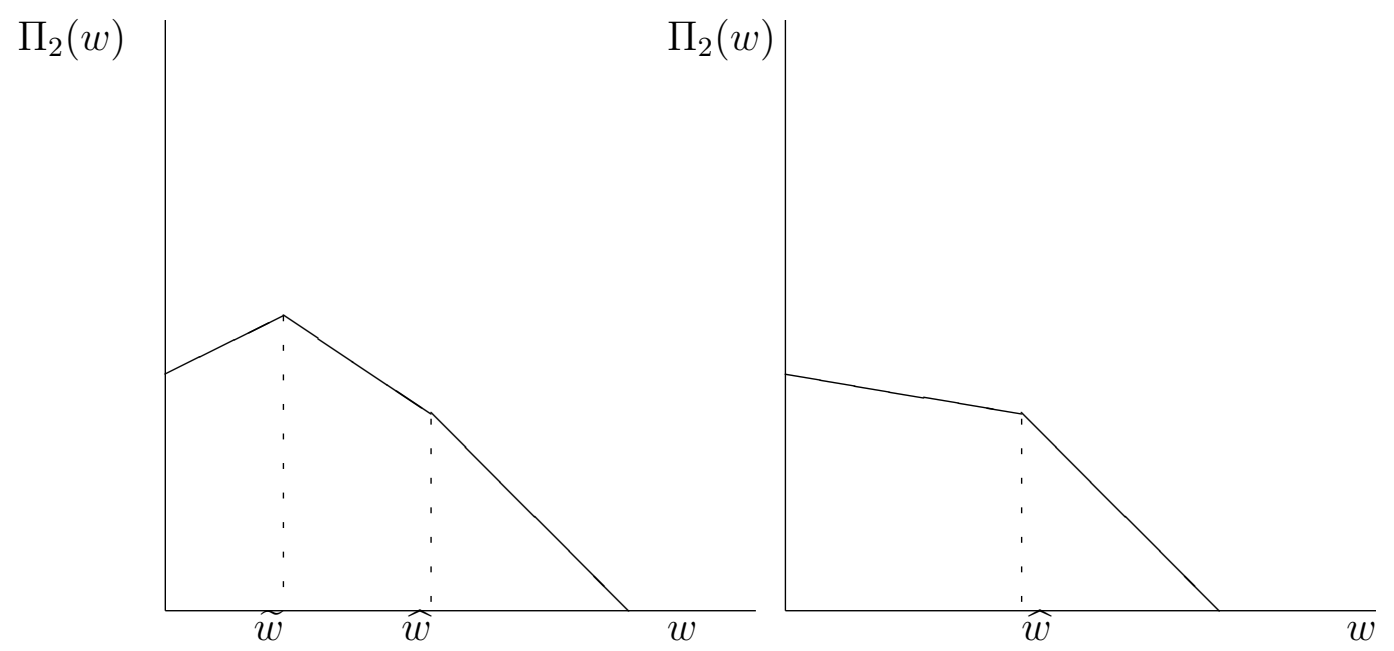

Figure 2: Value function for the second period problem

\subsection{The first period contract}

We solve for the optimal long-term contract taking as given the expected utility $w$ that the borrower is initially entitled to, and characterize in this way the entire Pareto frontier. As in the second period, the lender cannot costlessly observe the project outcome, but unlike the second period he can use dynamic incentives to reduce the severity of the information problem. The contract will specify monitoring probabilities $q: \Theta \times \Re_{+} \rightarrow[0,1]$, repayment functions $r: \Re_{+} \times \Theta \rightarrow \Re_{+}$and $R: \Re_{+} \times \Theta \times \Theta \rightarrow \Re_{+}$, and promised expect utilities $w: \Re_{+} \times \Theta \rightarrow \Re_{+}$and $W: \Re_{+} \times \Theta \times \Theta: \rightarrow \Re_{+}$, depending on whether the project realization has been monitored or not, to solve

$\Pi_{1}(w)=\max _{q_{i}, R_{i, j}, r_{i}, w_{i}, W_{i, j}} \sum_{i=H, L} p_{i}\left\{q_{i}\left[R_{i, i}+\delta \Pi_{2}\left(W_{i, i}\right)\right]+\left(1-q_{i}\right)\left[r_{i}+\delta \Pi_{2}\left(w_{i}\right)\right]-\gamma q_{i}\right\}$

$$
\text { s.t. } \left.\quad \sum_{i=L, H} p_{i}\left\{\theta_{i}+\left[q_{i}\left(\delta W_{i, i}-R_{i, i}\right]\right)+\left(1-q_{i}\right)\left(\delta w_{i}-r_{i}\right)\right]\right\}=w
$$

$$
\begin{gathered}
\theta_{i}+q_{i}\left(\delta W_{i, i}-R_{i, i}\right)+\left(1-q_{i}\right)\left(\delta w_{i}-r_{i}\right) \geq \theta_{i}+q_{j}\left(\delta W_{i, j}-R_{i, j}\right)+\left(1-q_{j}\right)\left(\delta w_{j}-r_{j}\right) \\
\text { if } r_{j} \leq \theta_{i} \quad \text { and } \quad \mathrm{i} \neq \mathrm{j}
\end{gathered}
$$




$$
0 \leq R_{i, j}, r_{i} \leq \theta_{i}, \quad 0 \leq w_{i}, W_{i, j} \leq \bar{w} \quad 0 \leq q_{i} \leq 1
$$

Notice that the promised utilities $W$ and $w$ now enter in the incentive compatibility constraint (14) in addition to the repayment functions $R$ and $r$, where $R_{i, j}$ and $W_{i, j}$ are the repayments and the future promised expected utilities when monitoring reveals the true income realization is $\theta_{i}$ and the lender announced to be $\theta_{j}$, while $r_{i}$ and $w_{i}$ are the repayment and the promised utility when the announced state is $\theta_{i}$ and the true realization is not verified.

Lemma 3. The optimal contract satisfies:

a. For $i \neq j$ it is optimal to set $R_{i j}=\theta_{i}$ and $W_{i, j}=0$.

b. $W_{i i} \geq w_{i}$.

c. The incentive compatibility constraint (14) is binding for $\theta_{i}=\theta_{H}$. In this case, $q_{H}=0$. Finally, the incentive compatibility (14) can be ignored for $\theta_{i}=\theta_{L}$.

d. $q_{L}=1$ if and only if $w=0$.

e. $w_{H} \geq W_{L L} \geq w_{L} ; R_{L L}=\theta_{L}$.

Using these results, we can solve for $r_{H}$ and $r_{L}$ in the promise keeping constraint and in the incentive compatibility constraint. The problem then consists in choosing a probability of monitoring $q_{L} \geq 0$ (we know $q_{L}<1$ except the case $w=0$ ) and promised expected utilities $0 \leq w_{H}, w_{L}, W_{L L} \leq \bar{w}$ to maximize the objective

$$
\begin{aligned}
p \theta_{H} & +(1-p) \theta_{L}-\gamma(1-p) q_{L}+\delta\left\{p\left(\Pi_{2}\left(w_{H}\right)+w_{H}\right)\right. \\
& \left.+(1-p)\left[q_{L}\left(\Pi_{2}\left(W_{L L}\right)+W_{L L}\right)+\left(1-q_{L}\right)\left(\Pi_{2}\left(w_{L}\right)+w_{L}\right)\right]\right\}-w
\end{aligned}
$$

subject to the relevant limited liability constraints on $r_{L}$

$$
\left(1-q_{L}\right)\left[\delta w_{L}+p\left(\theta_{H}-\theta_{L}\right)\right]+q_{L} \delta(1-p) W_{L L}-w \leq 0
$$

and the relevant limited liability constraint on $r_{H}$

$$
\delta w_{H}-\left(1-q_{L}\right)(1-p)\left(\theta_{H}-\theta_{L}\right)+q_{L} \delta W_{L L}-w \leq 0
$$


Equation (16) consists of the discounted sum of state-contingent expected aggregate surplus, where the current aggregate surplus is $p \theta_{H}+(1-p) \theta_{L}-$ $\gamma(1-p) q_{L}$. The objective is monotone increasing in each of the promised utilities $w_{H}, w_{L}$ and $W_{L L}$, whereas the role of the monitoring probability $q_{L}$ is not as straightforward. Indeed, a higher probability of monitoring reduces expected revenues, because monitoring costs increase, but it also moves some mass from the state corresponding to the promised utility $w_{L}$ to the one corresponding to $W_{L L}$, and thereby increases expected revenues given that $W_{L L} \geq w_{L}$ and $\Pi_{2}(w)+w$ is monotone increasing.

It is easy to see that the optimal solution would be to choose $q_{L}=0$, $w_{L}, w_{H}, W_{L L} \geq \widehat{w}$, but from (17) this is possible only if $w \geq p\left(\theta_{H}-\theta_{L}\right)+\delta \widehat{w}$, whereas for a smaller value of $w$ (17) must be binding and one of the conditions above cannot be met. Solving for the probability of monitoring we get

$$
q_{L}=\frac{p\left(\theta_{H}-\theta_{L}\right)+\delta w_{L}-w}{p\left(\theta_{H}-\theta_{L}\right)+\delta w_{L}-(1-p) \delta W_{L L}} .
$$

From Lemma $3, q_{L}<1$, which implies $w>(1-p) \delta W_{L L}$ and therefore $q_{L}$ is non-negative only for $p\left(\theta_{H}-\theta_{L}\right)+\delta w_{L}-w \geq 0$ and $p\left(\theta_{H}-\theta_{L}\right)+\delta w_{L}-(1-$ p) $\delta W_{L L}>0$. Therefore, the denominator in $\left(^{*}\right)$ is strictly positive. Replacing $q_{L}$ in (16) and in (18), the problem is differentiable everywhere but the two kinks $\widehat{w}$ and $\widetilde{w}$. Consider then the collection of restricted problems that we obtain when we restrict promised utilities to belong to one of the intervals where $\Pi_{2}(\cdot)$ is differentiable; let $\lambda_{1}^{k}$ and $\lambda_{2}^{k}$ be the multiplier associated with the boundary conditions on the promised utility $w_{k}$, let $\mu$ be the multiplier associated with (18), and $\lambda_{q}$ the one associated with the non-negativity of the monitoring probability. Linearity of the value function on these intervals makes it possible to write $\Pi_{2}\left(w_{k}\right)=A_{k}+\phi_{k} w_{K}$.

The first order conditions associated with the promised utilities are

$$
\left(1+\phi_{H}\right) \delta p_{H}+\lambda_{1}^{H}-\lambda_{2}^{H}-\delta \mu=0
$$

for $w_{H}$, while the promised utility $W_{L L}$ must satisfy

$$
\begin{aligned}
\delta K\left(w_{L}, W_{L L}\right) q_{L}\left\{(1-p) \delta\left[A_{L L}-A_{L}+w_{L}\left(\phi_{L L}-\phi_{L}\right)\right]-p_{L} \gamma\right. & \\
& \left.+\left(\theta_{H}-\theta_{L}+\delta w_{L}\right)\left(p_{H}\left(1+\phi_{L L}\right)-\mu\right)\right\}+\lambda_{1}^{L L}-\lambda_{2}^{L L}=0 \\
& \quad\left(F O C_{L L}\right)
\end{aligned}
$$


and finally $w_{L}$ must satisfy

$$
\begin{aligned}
\delta K\left(w_{L}, W_{L L}\right) & \left(1-q_{L}\right)\left\{\delta\left[A_{L L}-A_{L}+W_{L L}\left(\phi_{L L}-\phi_{L}\right)\right]-\gamma\right. \\
& \left.+\left(\theta_{H}-\theta_{L}+\delta W_{L L}\right)\left(p_{H}\left(1+\phi_{L}\right)-\mu\right)\right\}+\lambda_{1}^{L}-\lambda_{2}^{L}+\lambda_{q} \delta=0
\end{aligned}
$$

where $K\left(w_{L}, W_{L L}\right)$ in both $\left(F O C_{L L}\right)$ and $\left(F O C_{L}\right)$ is defined as

$$
K\left(w_{L}, W_{L L}\right)=\frac{(1-p)}{p\left(\theta_{H}-\theta_{L}\right)+\delta w_{L}-(1-p) \delta W_{L L}}>0 .
$$

Proposition 2. There are two thresholds $w^{*}$ and $w^{* *}$ (with $w^{*}<w^{* *}$ ) on the promised utility $w$ such that the optimal contract always involves the minimum feasible probability of monitoring $q_{L}$ if either $w<w^{*}$ or $w>w^{* *}$. For $w^{*} \leq w \leq w^{* *}$ instead, if the costs of verification $\gamma$ are not too high, the optimal probability of monitoring is strictly higher than its feasible lower bound.

Proof. The proof consists of a collection of results that all together characterize the entire Pareto frontier. In what follows, $q_{L}>(=) 0$ must be interpreted as $w_{L}>(=) p\left(\theta_{H}-\theta_{L}\right)-w$, moreover recall from before the definitions of $\widehat{w}=p\left(\theta_{H}-\theta_{L}\right)-\psi$ and $\widetilde{w}=\frac{p(0)}{p-p(0)} \psi$.

Observation 1: If $q_{L}>0$, then both $w_{L}, W_{L L} \leq \widehat{w}$.

Suppose first that $w_{L}>\widehat{w}$ and $q_{L}>0$. Then $\phi_{L}=\phi_{L L}=-1$ and $A_{L}=A_{L L}$, therefore from $\left(F O C_{L}\right)$ we have $\mu=-C_{1} \lambda_{2}^{L}-C_{2} \gamma<0$, for $C_{1}$ and $C_{2}$ positive numbers, which is a contradiction. Suppose now that only $W_{L L}>\widehat{w}$, while $w_{L} \leq \widehat{w}$. Form $\left(F O C_{L L}\right)$,

$$
\mu \leq(1-p) \frac{\delta\left(A_{L L}-A_{L}+w_{L}\left(\phi_{L L}-\phi_{L}\right)\right)-\gamma}{\theta_{H}-\theta_{L}+\delta w_{L}}
$$

which implies $\mu \geq 0$ only if $\gamma \leq \delta\left(A_{L L}-A_{L}-\delta w_{L}\left(\phi_{L L}-\phi_{L}\right)\right)$. Replacing both the upper bound for $\gamma$ and $\mu$ in $\left(F O C_{L}\right)$ we have

$$
\begin{gathered}
0=\left(F O C_{L}\right) \geq\left(1+\phi_{L}\right)\left(1-q_{L}\right)(1-p) \delta+\lambda_{1}^{L}-\lambda_{2}^{L}>\lambda_{1}^{L}-\lambda_{2}^{L} \\
\Rightarrow \lambda_{2}^{L}>0
\end{gathered}
$$


which implies $w_{L}=\widehat{w}$. But then $\gamma>0=\delta\left(A_{L L}-A_{L}-\delta w_{L}\left(\phi_{L L}-\phi_{L}\right)\right)$, which is a contradiction.

Observation 2: $w_{L}>\widetilde{w}$ only if $q_{L}=0$.

Suppose $w_{L}>\widetilde{w}$ and $q_{L}>0$. Sine we know that both $w_{L}$ and $W_{L L}$ have to be less or equal to $\widehat{w}$ when $q_{L}>0$, we can replace for $A_{L}=A_{L L}$ and $\phi_{L}=\phi_{L L}$ in $\left(F O C_{L}\right)$ and get an expression for $\mu$

$$
\begin{aligned}
\mu \leq & \left(p\left(\theta_{H}-\theta_{L}+\delta W_{L L}\right)\left(1+\phi_{L}\right)-\gamma\right) C_{1}+\lambda_{q} C_{2} \\
& \leq C_{1}\left\{-\gamma+(1-p) \gamma\left(1+\frac{\delta W_{L L}}{\theta_{H}-\theta_{L}}\right)\right\}+\lambda_{q} C_{2}<\lambda_{q} C_{2}=0
\end{aligned}
$$

where $C_{1}$ and $C_{2}$ are positive numbers, the first inequality comes from the definition of $\phi_{L}$ and the second one follows from the fact that $W_{L L} \leq \widehat{w}$.

Observation 3: If the constraint (18) is slack, then $w_{H} \geq \widehat{w}$ and $W_{L L}=\widehat{w}$. The first conclusion, $w_{H} \geq \widehat{w}$, follows immediately from $\left(\bar{F} O C_{H}\right)$. Regarding the second point, if $q_{L}=0$ we can assume w.l.o.g that $W_{L L}=\widehat{w}$, while we already know that $W_{L L} \leq \widehat{w}$ if $q_{L}>0$. Since (18) is slack, from equation $\left(F O C_{L L}\right)$ we have

$$
\begin{aligned}
0= & C_{1}\left\{p\left(1+\phi_{L L}\right)\left(\theta_{H}-\theta_{L}+\delta w_{L}\right)+(1-p)\left[\delta\left(A_{L L}-A_{L}+w_{L}\left(\phi_{L L}-\phi_{L}\right)\right)\right.\right. \\
& \left.-\gamma]+\lambda_{1}^{L L}-\lambda_{2}^{L L}\right\} \\
& \left.\geq C_{1}\left\{p\left(1+\phi_{L L}\right)\left(\theta_{H}-\theta_{L}+\delta w_{L}\right)-\gamma\right]+\lambda_{1}^{L L}-\lambda_{2}^{L L}\right\} \\
& \geq C_{1}\left\{-(1-p) \gamma+(1-p) \gamma\left(1+\frac{\delta w_{L}}{\theta_{H}-\theta_{L}}\right)+\lambda_{1}^{L L}-\lambda_{2}^{L L}\right\}>C_{1}\left(\lambda_{1}^{L L}-\lambda_{2}^{L L}\right)
\end{aligned}
$$

where $C_{1}$ is a positive number, the first inequality follows from the fact that $w_{L} \leq W_{L L}$ and $A_{L L}+x \phi_{L L} \geq A_{L}+x \phi_{L}$ if $x \leq W_{L L}$ and the second one comes from the definition of $\phi_{L L}$ and the last one from the non-negativity of $w_{L}$.

Observation 4: If the constraint (18) is slack, there exist a threshold $\widehat{\gamma}_{1}$ such that, if $\gamma>\widehat{\gamma}_{1}$, then either $w_{L}=0$ or $q_{L}=0$, if $\gamma \leq \widehat{\gamma}_{1}$ then $w_{L}=\widetilde{w}$. We already know $W_{L L}=\widehat{w}$ for (18) slack. Replacing it in $\left(F O C_{L}\right)$ and noticing that $A_{L L}+\widehat{w} \phi_{L L}=\theta_{L}$, and $A_{L}=1$ we get

$0=C_{1}\left\{p\left(1+\phi_{L}\right)\left(\theta_{H}-\theta_{L}+\delta \widehat{w}\right)-\gamma+\delta\left[\theta_{L}-1-\phi_{L} \widehat{w}\right]\right\}+C_{2}\left(\delta \lambda_{q}+\lambda_{1}^{L}-\lambda_{2}^{L}\right)$ 
Define $\widehat{\gamma}_{1}$ the solution to

$$
\widehat{\gamma}_{1}=F\left(\widehat{\gamma}_{1}\right)=p\left(1+\phi_{L}\left(\widehat{\gamma}_{1}\right)\left(\theta_{H}-\theta_{L}+\delta \widehat{w}\right)+\delta\left[\theta_{L}-1-\phi_{L} \widehat{w}\right)\right.
$$

Since $F(0)>0$ and $F^{\prime}(\gamma)<0$ there is a unique $\widehat{\gamma}_{1}$ satisfying the equation above. It follows that for $\gamma$ greater than such a threshold, either $\lambda_{q}>0$ or $\lambda_{L}^{1}>0$, and for $\gamma<\widehat{\gamma}$, if $q_{L}>0$ then $\lambda_{2}^{L}>0$ and $w_{L}=\widetilde{w}$

Observation 5: If the limited liability constraint (18) is binding, $w_{H}<\widetilde{w}$ only if $w_{L}=W_{L L}=0$. If $w_{H} \geq \widetilde{w}$ there exist thresholds $\widehat{\gamma}_{2}, \widehat{\gamma}_{3}, \widehat{\gamma}_{4}$ such that

1. If $\gamma>\widehat{\gamma_{2}} \Rightarrow \widetilde{w}<W_{L L} \leq \widehat{w}$ only if $w_{L}=0$ or $q_{L}=0$, and $w_{H}=\widehat{w}$.

If $\gamma \leq \widehat{\gamma}_{2} \Rightarrow w_{L}=\widetilde{w}$ and $w_{H}=\widehat{w}$ if $W_{L L}>\widetilde{w}$.

2. If $\gamma>\widehat{\gamma}_{3} \Rightarrow w_{H}<\widehat{w}$ only if $w_{L}=0$ or $q_{L}=0$.

If $\gamma \leq \widehat{\gamma}_{3} \Rightarrow w_{L}=\widetilde{w}$ if $w_{H} \geq \widetilde{w}$.

3. if $\gamma>\widehat{\gamma}_{4} \Rightarrow w_{H}<\widehat{w}$ only if $W_{L L}=0$.

If $\gamma \leq \widehat{\gamma}_{4} \Rightarrow W_{L L}=\widetilde{w}$ if $w_{H} \geq \widetilde{w}$.

Solve for $\mu$ in $\left(F O C_{L}\right)$,

$$
\begin{aligned}
\mu=p\left(1+\phi_{L}\right) & +\frac{\delta\left(A_{L L}-A_{L}+W_{L L}\left(\phi_{L L}-\phi_{L}\right)\right)-\gamma}{\theta_{H}-\theta_{L}+\delta W_{L L}} \\
& +\frac{\lambda_{1}^{L}-\lambda_{2}^{L}+\lambda_{q} \delta}{K \delta(1-q)\left(\theta_{H}-\theta_{L}+\delta W_{L L}\right)}
\end{aligned}
$$

and replace it in $\left(F O C_{L L}\right)$

$$
\begin{aligned}
\frac{q_{L} \delta}{\theta_{H}-\theta_{L}+\delta W_{L L}}\left\{\frac{\lambda_{2}^{L}-\lambda_{1}^{L}-\lambda^{q} \delta}{(1-q) \delta}\left(\theta_{H}-\theta_{L}+\delta w_{L}\right)+\right. \\
\left.(1-p)\left[\left(\phi_{L L}-\phi_{L}\right)\left(\theta_{H}-\theta_{L}\right)-\delta\left(A_{L L}-A_{L}\right)+\gamma\right]\right\}+\lambda_{1}^{L L}-\lambda_{2}^{L L}=0 \\
\left(F O C_{L L}^{\prime}\right)
\end{aligned}
$$

and in $\left(F O C_{H}\right)$

$$
\begin{aligned}
p\left(1+\phi_{H}\right)+\frac{\lambda_{1}^{H}-\lambda_{2}^{H}}{\delta}=p\left(1+\phi_{L}\right) & +\frac{\delta\left(A_{L L}-A_{L}+W_{L L}\left(\phi_{L L}-\phi_{L}\right)\right)-\gamma}{\theta_{H}-\theta_{L}+\delta W_{L L}} \\
& +\frac{\lambda_{1}^{L}-\lambda_{2}^{L}+\lambda_{q} \delta}{K \delta(1-q)\left(\theta_{H}-\theta_{L}+\delta W_{L L}\right)}\left(F O C_{H}^{\prime}\right)
\end{aligned}
$$


Consider the first point above: ignoring for now the multipliers, define in the expression in brackets in $\left(F O C_{L L}^{\prime}\right)$ the threshold $\widehat{\gamma}_{2}$ as the solution to

$$
\widehat{\gamma}_{2}=F\left(\widehat{\gamma}_{2}\right)=\left(\phi_{L}\left(\widehat{\gamma}_{2}\right)-\phi_{L L}\left(\widehat{\gamma}_{2}\right)\right)\left(\theta_{H}-\theta_{L}\right)+\delta\left(A_{L L}\left(\widehat{\gamma}_{2}\right)-A_{L}\left(\widehat{\gamma}_{2}\right)\right)
$$

Given $W_{L L} \geq w_{L}$, it is easy to see that $F(0)=0$ and $F^{\prime}(\gamma)<0$. Therefore there exist a unique $\widehat{\gamma}_{2}$ such that $\widehat{\gamma}_{2}=F\left(\widehat{\gamma}_{2}\right)$, with the property that the term in brackets in $\left(F O C_{L L}^{\prime}\right)$ is positive for $\gamma$ bigger that it and it is negative for $\gamma$ smaller. Considering now the multipliers, it is straightforward that if $\gamma \leq \widehat{\gamma}_{2}$, then either $q_{L}=0$ or $\lambda_{1}^{L}>0$ or $\lambda_{2}^{L L}>0$, if $\gamma>\widehat{\gamma}_{2}$ then either $\lambda_{2}^{L}>0$ or $\lambda_{1}^{L L}>0$ (if $q_{L}>0$ ).

If $W_{L L}>\widetilde{w}$, then from $\left(F O C_{L L}^{\prime}\right)$

$$
\begin{aligned}
\frac{\lambda_{1}^{L}-\lambda_{2}^{L}+\lambda_{q} \delta}{K \delta(1-q)\left(\theta_{H}-\theta_{L}+\delta W_{L L}\right)} & \leq(1-p)\left[p\left(\theta_{H}-\theta_{L}\right)+\delta w_{L}-(1-p) \delta W_{L L}\right] \\
& \times \frac{\left(\phi_{L L}-\phi_{L}\right)\left(\theta_{H}-\theta_{L}\right)-\delta\left(W_{L L}-A_{L}\right)+\gamma}{\left(\theta_{H}-\theta_{L}+\delta w_{L}\right)\left(\theta_{H}-\theta_{L}+\delta W_{L L}\right)}
\end{aligned}
$$

that replaced in $\left(F O C_{L}^{\prime}\right)$ gives

$$
\mu \leq p\left(1+\phi_{L L}\right)+(1-p) \frac{\delta\left(A_{L L}-A_{L}+\left(\phi_{L L}-\phi_{L}\right) \delta w_{L}\right)-\gamma}{\theta_{H}-\theta+\delta w_{L}}
$$

If $\gamma>\widehat{\gamma}$ and $q>0$, we get

$$
\begin{aligned}
\mu & \leq p\left(1+\phi_{L L}\right)+(1-p) \frac{\delta\left(A_{L L}-A_{L}+\left(\phi_{L L}-\phi_{L}\right) \delta w_{L}\right)-\widehat{\gamma}_{2}}{\theta_{H}-\theta+\delta w_{L}} \\
& \leq p\left(1+\phi_{L L}\right)+(1-p)\left(\phi_{L L}-\phi_{L}\right)<p\left(1+\phi_{L L}\right)
\end{aligned}
$$

If instead $\gamma \leq \widehat{\gamma}$ and $q>0$ then $w_{L}=\widetilde{w}$ and $A_{L L}-A_{L}+\widetilde{w}\left(\phi_{L L}-\phi_{L}\right)=0$, therefore

$$
\mu \leq p\left(1+\phi_{L L}\right)-(1-p) \gamma<p\left(1+\phi_{L L}\right)
$$

In both cases $\left(F O C_{H}^{\prime}\right)$ gives $w_{H}=\widehat{w}$, concluding the first part. Consider now the remaining part of the proposition and let $w_{L}, W_{L L} \leq \widetilde{w}$. First of all notice from $\left(F O C_{L L}^{\prime}\right)$ that if $W_{L L}<\widetilde{w}$, then either $q_{L}=0$ or $w_{L}=0$. Replace than $w_{L}=0$ and solve $\left(F O C_{L L}^{\prime}\right)$ for $\lambda_{1}^{L}$; finally replace such a value in $\left(F O C_{H}^{\prime}\right)$

$$
\gamma=\frac{\lambda_{1}^{H}-\lambda_{2}^{H}}{\delta}+\frac{p\left(\phi_{L}-\phi_{H}\right)\left(\theta_{H}-\theta_{L}\right)}{1-p}
$$




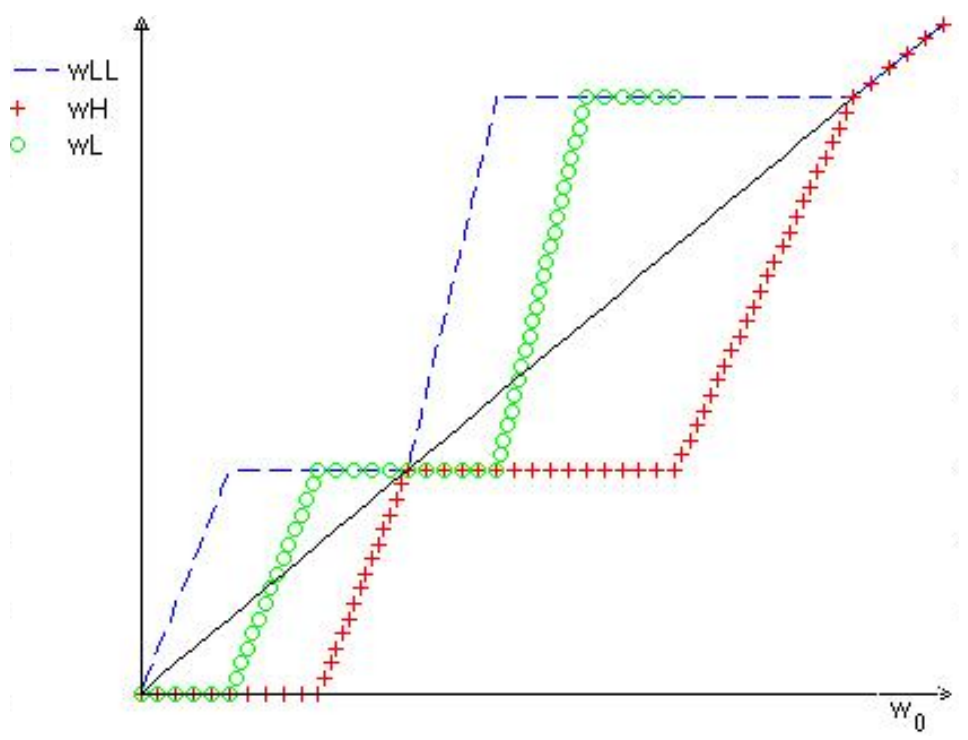

Figure 3: Low Monitoring Cost

Define $\widehat{\gamma}_{3}=\frac{p\left(\phi_{L}-\phi_{H}\right)\left(\theta_{H}-\theta_{L}\right)}{1-p}$ and the second conclusion follows.

Observe now, in $\left(F O C_{L L}^{\prime}\right)$, that for $w_{L}>0$ it must be $W_{L L}=\widetilde{w}$. Replace then $W_{L L}=\widetilde{w}$ in $\left(F O C_{L}^{\prime}\right)$ and $\left(F O C_{H}^{\prime}\right)$ and get

$$
p\left(\phi_{L}-\phi_{H}\right)\left(\theta_{H}-\theta_{L}+\delta \widetilde{w}\right)=\gamma+\frac{\lambda_{1}^{H}-\lambda_{2}^{H}}{\delta}
$$

Define than $\left.\widehat{\gamma}_{4}=p\left(\phi_{L}-\phi_{H}\right) \theta_{H}-\theta_{L}+\delta \widetilde{w}\right)$ and the conclusion is immediate. Let in the end be $w_{H}<\widetilde{w}$. In $\left(F O C_{L}^{\prime}\right)$ it's straightforward that $w_{L}=0$ and solving for $\lambda_{1}^{L}$ in it and substitute it in $\left(F O C_{L L}^{\prime}\right)$ to get, for $C_{\text {! }}$ a positive number,

$$
\begin{gathered}
C_{1}\left\{-(1-p) \frac{\theta_{H}-\theta_{L}+\delta W_{L L}}{K}\right\}+\lambda_{1}^{L L}-\lambda_{2}^{L L}=0 \\
\Rightarrow W_{L L}=0
\end{gathered}
$$

\subsubsection{Discussion}

Solving for the optimal long-term contract is equivalent to choosing how much to rely on dynamic incentives and how much to rely on current mon- 


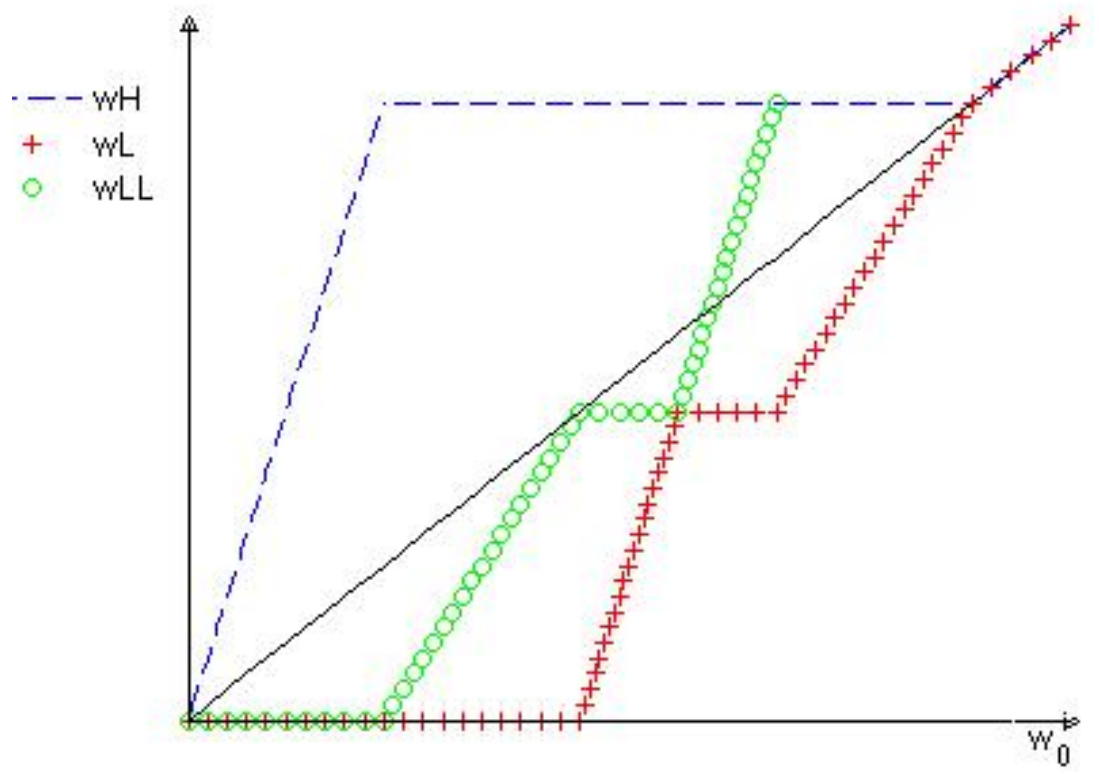

Figure 4: Medium Monitoring Cost

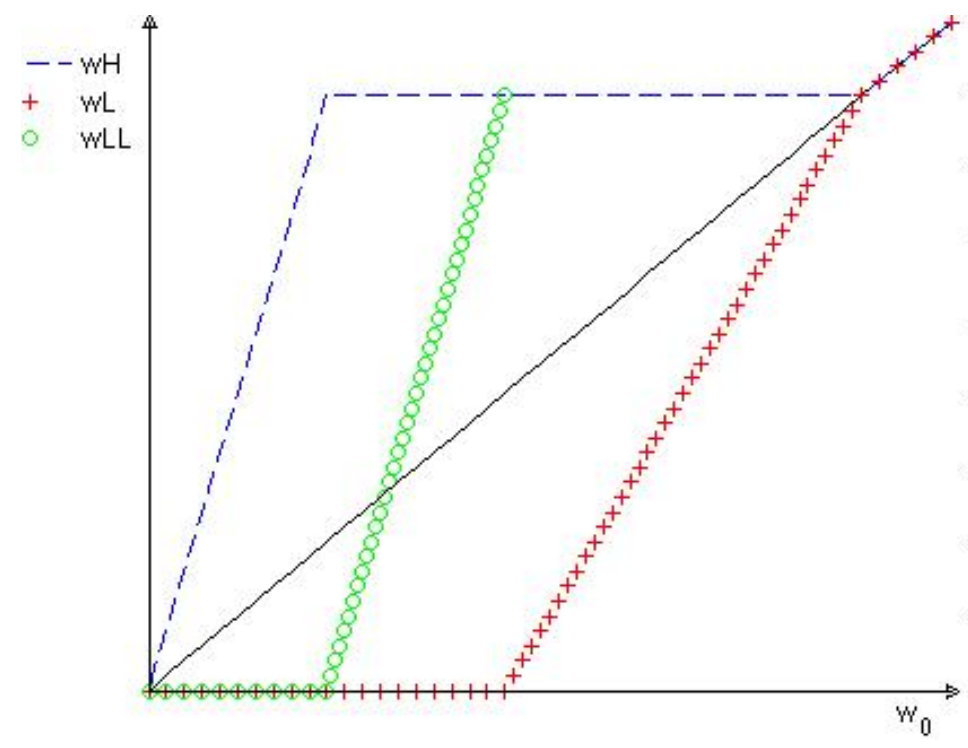

Figure 5: High Monitoring Cost 
itoring to satisfy the incentive compatibility constraint (14) and the limited liability constraints on repayments and promised utilities, while taking as given the promised expected utility $w$ of the borrower. The solution depends on $w$, because it is the promised expected utility $w$ that determines the set of active constraints, the set of feasible repayments and ultimately benefits and costs of the two instruments. Figures 3, 4, and 5 show the relationship between the initial promised utility to the borrower and the state-contingent promised utilities after the first-period investment is realized respectively for low, medium, and high monitoring costs.

In general, as the promised utility $w$ gets smaller, satisfying the incentive compatibility constraint (14) requires either the probability of monitoring to increase or the promised utility $w_{L}$ to decrease. Both instruments are costly to the lender: the cost of increasing the intensity of monitoring is $(1-p) \gamma$, whereas the cost of reducing $w_{L}$ consists in a lower aggregate surplus in the second period when the project yields a low realization in the first period. Formally, the loss of aggregate surplus is embedded into the slope of the value function $\Pi_{2}(\cdot)$. The verification technology is therefore a static instrument used to align current incentives with the forward-looking value of preserving a higher future expected joint surplus.

Proposition 2 proves that monitoring is not valuable if termination in the second period is never threatened by the equilibrium contract or if, at the other extreme, termination is unavoidable irrespectively of the first period realization. In both cases, the provision of dynamic incentives crowds out monitoring. In the first circumstance, this is because the loss of aggregate surplus associated with a lower $w_{L}$ is limited, by linearity of both the utility functions and the technology, to the cost of a higher intensity of verification at time $t=2$ contingent on a bad realization in the first period, and the potential gains associated with increased monitoring are not enough to cover its cost. Then if $w$ is relatively high, the most efficient way to induce the borrower to truthfully report the realization $\theta_{H}$ is simply to reduce the promised utility $w_{L}$ up to the point that $q_{L}=0$ in $(*)$. The second period contract will exhibit memory, the borrower will be entitled to a different expected utility and to a different fraction of the second period aggregate surplus depending on first period realization. In the second case, which happens for small values of $w$, the expected future gains associated with monitoring vanish completely because $w_{L}, W_{L L}$, and $w_{H}$ must all involve a positive probability of termination, the limited liability constraint must be necessarily binding, and because of the linearity of $\Pi_{2}(\cdot)$ in the lower interval. Indeed, in this case additional monitoring is associated with an increase in expected aggregate surplus conditional on a low realization and an identical decrease in expected aggregate surplus conditional on a high realization, so the increase in monitoring just 
represents a net social loss.

For intermediate values of the promised expected utility $w$, the expected future gains associated with monitoring can possibly cover its costs: it is still possible to save on the intensity of monitoring (and therefore on its costs) by decreasing $w_{L}$, but after a low realization in the first period the contractual conditions would have to deteriorate up to the point that termination becomes the lender best strategy. In this scenario, monitoring the borrower's project relaxes the information problem and allows the lender to offer a contract that doesn't depend on the first period realization (at least, since we are allowing for randomization over strategies, the dependence is relatively weaker), continue the relationship in any state at the end of $t=1$, and therefore avoid a costly termination of the contract. Whether monitoring is indeed preferred to terminating the contract depends contemporaneously on the cost $\gamma$ and on the state variable $w$. The dependence on $\gamma$ is obvious: the higher this is the less attractive the monitoring option is. The reason why the solution should change with the state variable $w$ is a bit more subtle: the margins between the provision of dynamic incentives and monitoring change with it through the limited liability constraints and the slopes $\phi_{L}, \phi_{L L}$ and $\phi_{H}$ of the value function $\Pi_{2}(\cdot)$. This explains why there is more than one threshold on $\gamma$, defined for disjoint intervals on the set of promised utilities $w$ the borrower can possibly be entitled to.

\section{Conclusions}

We studied the optimal contract between a lender and a borrower when the borrower has private information and the lender can resort to monitoring and dynamic incentives in the form of higher future promised utility and termination. We have characterized the entire efficiency frontier. The results show how monitoring and dynamic incentives can interact, and indicate how monitoring, although socially costly, is useful in a dynamic relationship. Specifically, the model shows that periodic costly monitoring can perform the role of keeping a contractual relation healthy enough to prevent inefficient liquidation.

There are some extensions to the model, and perhaps the most desirable would be to study the problem in an infinite horizon setting. Preferences also play a pivotal role, and the extension of the framework to risk averse agents is important, especially because often default is closely linked to the provision of insurance.

There are also potentially interesting applications. In the previous litera- 
ture, for example, Smith and Wang [15] applies a simple dynamic costly state verification environment to the problem of deposit insurance and lender-oflast-resort loans. Another example is Krasa, Sharma, and Villamil [9], which studies the effects of costly enforcement (interpreted as a court) on firm finance. Our model potentially lends itself to the analysis of situations where monitoring, dynamic incentives, and the threat of termination play a role in a bilateral relationship in which recourse to other kinds of enforcement is too costly or unavailable. For example, international loans programs such as the ones undertaken by international organizations, or venture capital financing. 


\section{APPENDIX}

\section{Proof of Lemma 1}

Observe first that if $q_{L}>0$, the truth-telling constraint (8) for a high realization is binding:

$$
q_{L} \theta_{H}+\left(1-q_{L}\right) r_{L}=q_{H} R_{H, H}+\left(1-q_{H}\right) r_{H}
$$

because if this was not the case, slightly decrease the probability of monitoring to $q_{L}^{\prime}=q_{L}-\epsilon$ and adjust the repayment to $r_{L}^{\prime}=r_{L}+\epsilon \frac{R_{L, L}-r_{L}}{1-q_{L}+\epsilon}$. No constraint is violated and the expected revenues increase by saving on monitoring costs.

Second, if $q_{L}=0$, then also $q_{H}=0$. By contradiction, if $q_{L}=0$ and $q_{H}>0$ it follows that $r_{L} \geq q_{H} R_{H, H}+\left(1-q_{H}\right) r_{H}$ which substituted into the (9) implies that the contract is compatible with effort $e=1$. Consider now a new contract with $q_{H}^{\prime}=0$ and $r_{H}^{\prime}=r_{L}^{\prime}=p(1)\left(q_{H} R_{H, H}+\left(1-q_{H}\right) r_{H}\right)+(1-p(1)) r_{L}$. Such a contract still implement the effort $e=1$ and saving on verification costs dominates the original one.

Third, no monitoring is performed in the high state: $q_{H}=0$

Suppose by contradiction that $q_{H}>0$. It follows from the previous lemmas that it must be also that $q_{L}>0$ and $q_{L} \theta_{H}+\left(1-q_{L}\right) r_{L}=q_{H} R_{H, H}+\left(1-q_{H}\right) r_{H}$. Consider than a new contract with $q_{H}^{\prime}=0$ and $r_{H}^{\prime}=q_{H} R_{H, H}+\left(1-q_{H}\right) r_{H}$. The only constraint we need to check is the truth-telling for the low state since none else has changed by construction:

$$
\begin{gathered}
q_{H}^{\prime} \theta_{L}+\left(1-q_{H}^{\prime}\right) r_{H}^{\prime}=r_{H}^{\prime}=q_{H} R_{H, H}+\left(1-q_{H}\right) r_{H} \\
=q_{L} \theta_{H}+\left(1-q_{L}\right) r_{L}>q_{L} R_{L, L}+\left(1-q_{L}\right) r_{L}
\end{gathered}
$$

where the third equality follows from the binding truth-telling constraint of the original problem and the last inequality follows from the limited liability constraint.

Finally from the steps above, to show that the truth-telling constraint (8) is binding for a high realization $\theta_{H}$ and it can be ignored for a low realization $\theta_{L}$, it is sufficient to consider 2 cases: $\left(q_{L}=0, q_{H}=0\right)$ and $\left(q_{L}>0, q_{H}=0\right)$. In the first case both constraints are binding and $r_{H}=r_{L}$, while in the second case the constraint for $\theta_{H}$ is binding so $r_{H}=q_{L} \theta_{H}+\left(1-q_{L}\right) r_{L}$ and the constraint for the low realization is slack. 


\section{Proof of Lemma 3}

[a.] Observe that $R_{i j}$ and $W_{i j}$ do not enter, for $i \neq j$, neither in the objective function (12) nor in the promise keeping constraint (13). Suppose then that either $W_{i j}>0$ or $R_{i j}<\theta_{i}$ for some $i$, and $q_{i}>0$. Decrease $W_{i j}$ to $W_{i j}^{\prime}=W_{i j}-\epsilon$ and/or increase $R_{i j}$ to $R_{i j}^{\prime}=R_{i j}+\epsilon$. At the same time decrease $q_{i}$ to $q_{i}^{\prime}=q_{i}-\alpha$ and change $R_{i i}$ to $R_{i i}^{\prime}=\alpha\left(R_{j i i}-r_{i}\right) /\left(q_{i}-\alpha\right)$ and $W_{i i}$ to $W_{i i}^{\prime}=W_{i i}+\alpha\left(w_{i}-W_{i i}\right) /\left(q_{j}-\alpha\right)$ if $W_{i i}<w_{i}$ or $w_{i}$ to $w_{i}^{\prime}=$ $w_{i}+\alpha\left(W_{i i}-w_{j}\right) /\left(q_{i}-\alpha\right)$ if $W_{i i} \geq w_{i}$. In any case case the promise keeping constraint and the incentive compatibility for $\theta_{j}$ are satisfied by construction, and for $\alpha$ small enough also the incentive compatibility for $\theta_{i}$ is still satisfied. The lender expected revenues are,

$$
\Pi_{1}^{\prime}(w)=\Pi_{1}(w)+\alpha\left[\Pi_{2}\left(w_{i}\right)-\Pi_{2}\left(W_{i i}\right)+\phi_{i}\left(W_{i i}-w_{i}\right)\right]+\alpha p_{i} \gamma>\Pi_{1}(w)
$$

if $w_{i} \leq W_{i i}$ and

$$
\begin{aligned}
\Pi_{1}^{\prime}(w)= & \Pi_{1}(w)+\alpha\left[\Pi_{2}\left(w_{i}\right)-\Pi_{2}\left(w_{i i}\right)+\phi_{i i}\left(w_{i}-W_{i i}\right)\right]+\alpha p_{i} \gamma \\
& \geq \Pi_{1}(w)+\alpha\left[\Pi_{2}\left(w_{i}\right)-\Pi_{2}\left(w_{i i}\right)+\phi_{i}\left(w_{i}-W_{i i}\right)\right]+\alpha p_{i} \gamma \\
& >\Pi_{1}(w)
\end{aligned}
$$

if $W_{i i}<w_{i}$, where $\phi_{x}$ is the slope of $\Pi_{2}(x)$ and all the inequalities follow from the fact that $\phi_{x} \geq \phi_{y}$ for $x \leq y$.

[b.] Suppose $q_{i}>0$ and for $W_{i i}<w_{i}$, define $W_{i i}^{\prime}=w_{i}^{\prime}=q_{L} W_{i i}+(1-$ $\left.q_{L}\right) w_{i}$. This satisfies all constraints and by weak concavity of $\Pi_{2}(\cdot)$ expected revenues weakly increase. If the expected revenues don't change it should be that $W_{i i}$ and $w_{i}$ belong to the same interval and therefore $\Pi_{2}(x)=A_{L}+x \phi_{L}=$ $A_{L L}+x \phi_{L L}$ for any $w_{L} \geq x \geq w_{L L}$. Define then $q_{i}^{\prime}=q_{i}-z, w_{i}^{\prime \prime}=w_{i}^{\prime}-\alpha$ and $W_{i i}^{\prime \prime}=W_{i i}^{\prime}+\alpha\left[1 /\left(q_{i}-z\right)-1\right]$. and $R_{i i}^{\prime}=R_{i i}+\alpha\left(R_{i i}-r_{i}\right) /\left(q_{i}-\alpha\right)$. The promise keeping constraint is satisfied by construction and for $\alpha$ and $z$ sufficiently small all other constraints are also satisfied. The expected revenues of the new contract are $\Pi^{\prime}(w)=\Pi(w)+\alpha p_{i} \gamma$, that contradicts the optimality of the original contract.

Replace, in what follows, $R_{i j}=\theta_{i}$ and $W_{i j}=0$ in the incentive compatibility constraint (14).

[c.] Suppose that (14) is slack for $\theta_{i}=\theta_{H}$. If $q_{L}>0$ define then $q_{L}^{\prime}=q_{L}-\epsilon, r_{L}^{\prime}=r_{L}+\epsilon\left(R_{L L}-r_{L}\right) /\left(1-q_{L}+\epsilon\right)$ and $w_{L}^{\prime}=w_{L}+\epsilon\left(w_{L L}-\right.$ $\left.w_{L}\right) /\left(1-q_{L}+\alpha\right)$. It follows that the promise keeping constraint (13) and the incentive compatibility (14) for $\theta_{L}$ are satisfied by construction, and for 
$\epsilon$ sufficiently small (14) is still satisfied for $\theta_{H}$. The expected revenues will change to

$$
\begin{aligned}
\Pi_{1}^{\prime}(w) & =\Pi_{1}(w)+\alpha\left[\Pi_{2}(w L)-\Pi_{2}\left(W_{L L}+\phi_{L}\left(W_{L L}-w_{L}\right)\right]+(1-p) \gamma \epsilon\right. \\
& \geq \Pi_{1}(w)+(1-p) \gamma \epsilon>\Pi_{1}(w)
\end{aligned}
$$

where the weak inequality follows from the fact that $\phi_{L} \geq \phi_{L L}$ since $w_{L} \leq$ $W_{L L}$. If instead $q_{L}=0$, first observe it must be that $q_{H}=0$, otherwise defining $q_{H}^{\prime}=0, r_{H}^{\prime}=r_{L}^{\prime}$ and $w_{H}^{\prime}=w_{L}^{\prime}$ all constraints would be satisfied, monitoring costs would decrease and by the week concavity of $\Pi_{2}$ expected revenues would increase. Since $q_{H}=q_{L}=0$, if $r_{H} \leq \theta_{L}$ it follows that (14) must be binding by definition for both $\theta_{L}$ and $\theta_{H}$. If instead $r_{H}>\theta_{L}$ and (14) is slack for $\theta_{H}$, either $w_{L}$ and $w_{H}$ belong to the same interval, in which case we can consider without loss of generality a new contract with $r_{L}^{\prime}=r_{H}^{\prime}=p r_{H}+(1-p) r_{L}$ and $w_{H}^{\prime}=w_{L}^{\prime}=p w_{H}+(1-p) w_{L}$, or $w_{H}$ and $w_{L}$ belong to different interval (with $w_{H}>w_{L}$ ), therefore defining $w_{H}=w_{H}-\epsilon$ and $w_{L}=w_{L}+\epsilon p /(1-p)$ strictly increases revenues given that $\phi_{H}<\phi_{L}$.

Suppose now $q_{H}>0$. Define $q_{H}^{\prime}=0$ and $r_{H}^{\prime}=q_{H} R_{H H}+\left(1-q_{H}\right) r_{H}$. Expected revenues increase because of weak concavity of $\Pi_{2}(\cdot)$ and the lower monitoring costs. The promise keeping constraint is satisfied for $\theta_{H}$, what is left to see whether (14) is also satisfied for $\theta_{L}$ :

$$
\begin{aligned}
& \theta_{L}-\delta w_{H}-r_{H}=\theta_{L}-q_{L} \theta_{H}-\left(1-q_{L}\right) r_{L}+\delta\left(1-q_{L}\right) w_{L} \\
& \leq \theta_{L}-q_{L} R_{L L}-\left(1-q_{L}\right) r_{L}+\delta\left(1-q_{L}\right) w_{L}+\delta q_{L} W_{L L}
\end{aligned}
$$

where the inequality follows from the limited liability constraint on $R_{L L}$ and $W_{L L}$.

[d.] The if part is very easy: from the promise keeping constraints and the limited liability, if $w=0$ and $q_{L}<1$, it must be $w_{H}=0, r_{H}=\theta_{H}, w_{L}=0$ and $r_{L}=\theta_{L}$. The incentive compatibility constraint would look $\left(1-q_{L}\right) \theta_{H}=$ $\left(1-q_{L}\right) \theta_{L}$ which can be satisfied only for $q_{L}=1$. To prove the only if part, suppose that $q_{L}=1$ but $w>0$. From (14) it must be that $r_{H}=\theta_{H}$ and $w_{H}=0$. Increase first $w_{H}$ to $w_{H}^{\prime}=(1-p) W_{L L}$ and decrease $W_{L L}$ to $W_{L L}^{\prime}=(1-p) W_{L L}$. In such a way the promise keeping constraint (13) is satisfied by construction, revenues weakly increase(they increase if $W_{L L}$ did not belong to the first interval, they stay the same otherwise) and the incentive compatibility constraint (14) becomes slack. Define then $w_{L}^{\prime}=$ $W_{L L}^{\prime}, r_{L}^{\prime}=R_{L L}$ and decrease $q_{L}$ to $q_{L}^{\prime}$ that makes (14) bind:

$$
\delta w_{H}^{\prime}-\theta_{H}=q_{L}^{\prime}\left(\delta w_{L}^{\prime}-r_{L}^{\prime}\right)-q_{L}^{\prime} \theta_{H}
$$


Since the $L H S<R H S$ for $q_{L}=0$ and $L H S>R H S$ for $q_{L}=1$, such a $q_{L}^{\prime}<q_{L}$ always exist, and it follows that the expected revenues strictly increase by saving on monitoring costs, with the promise keeping constraint (13) still satisfied since it doesn't depend on $q_{L}$ given $W_{L L}^{\prime}=w_{L}^{\prime}$.

[e.] If $q_{L}=0$, we can assume without loss of generality that $w_{L}=W_{L L}$. Then if $w_{L}>w_{H}$, define $w_{L}^{\prime}=w_{H}^{\prime}=p w_{H}+(1-p) w_{L}$ and $r_{H}^{\prime}=r_{L}^{\prime}=$ $p r_{H}+(1-p) r_{L}$. All constraints are easily satisfied and by weak concavity of $\Pi_{2}$ expected revenues weakly increase.

If $q_{L}>0$, suppose $W_{L L}>w_{H}$. Then slightly increase $w_{H}$ and decrease $W_{L L}$ such that $p w_{H}^{\prime}+(1-p)(1-q) W_{L L}^{\prime}$ doesn't change. The promise keeping constraint is satisfied while the incentive compatibility constraint becomes slack. We know from before that $W_{L L} \geq w_{L}$. If $w_{L}=W_{L L}$ decrease the probability of monitoring $q_{L}$ to make the incentive compatibility constraint bind and adjust $R_{L L}$ to leave the promise keeping constraint unchanged. Expected revenues will increase by weak concavity of $\Pi_{2}$ and because of the lower monitoring intensity $q_{L}$. If instead $W_{L L}>w_{L}$ then for a small initial change $t$ is still true that $W_{L L}^{\prime}>w_{L}$. Then increase $w_{L}$ to make the incentive compatibility bind and decrease $W_{L L}^{\prime}$ to leave the promise keeping constraint binding. This will increase the revenues because of (weak) concavity of $\Pi_{2}$, unless $w_{L}, w_{H}$ and $W_{L L}$ all belong to the same interval. But if all the three belonged to the same interval then we could increase $w_{H}$ and decrease $q_{L}$ to leave the promise keeping constraint binding, and then either decrease $W_{L L}$ or increase $w_{L}$ to leave the promise keeping constraint unchanged and expected revenues would decrease because of the lower monitoring intensity.

Suppose now that $R_{L L}<\theta_{L}$. Notice the following two things: first, neither $W_{L L}$ nor $R_{L L}$ enter the incentive compatibility constraint. Second it must be that $W_{L L} \geq \widehat{w}$, where $\widehat{w}$ is the level of the promised utility $w_{2}$ such that no monitoring is carried out in the second period, otherwise by increasing both $R_{L L}$ and $W_{L L}$ to leave the promise keeping constraint satisfied, it would be possible to increase expected revenues.

If $R_{L L}<\theta_{L}$, it must be that $w_{L}<\widehat{w}, w_{H}=\bar{w}$ and $r_{H}=0$, where $\bar{w}$ is the highest utility the lender can promise to the borrower in the second period. Indeed, if $w_{L}<\widehat{w}$ and $w_{H}<\bar{w}$, define a new contract with $w_{H}^{\prime}=w_{H}+\epsilon$, $w_{L}^{\prime}=w_{L}+\epsilon /\left(1-q_{L}\right)$ and $R_{L L}^{\prime}=R_{L L}+\delta \epsilon /\left[q_{L}(1-p)\right]$. The incentive compatibility constraint and the promise keeping constraint are satisfied by the way the new contract is defined and for $\epsilon$ sufficiently small the limited liability constraints are also satisfied. Expected revenues would change to $\Pi(w)+\delta \epsilon\left(1+p \phi_{H}+(1-p) \phi_{L}\right)>\Pi(w)$ since both $\phi_{L}>-1$ and $\phi_{H} \geq-1$. If instead $w_{L}<\widehat{w}$ and $r_{H}>0$ define a new contract with $r_{H}^{\prime}=r_{H}-\epsilon$, 
$w_{L}^{\prime}=w_{L}+\epsilon /\left[\delta\left(1-q_{L}\right)\right]$ and $R_{L L}^{\prime}=R_{L L}+\epsilon /\left[(1-p) q_{L}\right]$ all constraints are satisfied and the new revenues are $\Pi(w)+\epsilon\left(-p+(1-p) \phi_{L}+1\right)>\Pi(w)$ given $\phi_{L}>-1$. It follows from the incentive compatibility constraint that if $r_{H}=0$ and $w_{H}=\bar{w}$, the only feasible probability of monitoring is $q_{L}=0$ with $w_{L}=\bar{w}$ and $r_{L}=0$.

If instead $w_{L} \geq \widehat{w}$ and given we know from before $w_{L L} \geq w_{L}$, it must hold also in this case $w_{H}=\bar{w}$ and $r_{H}=0$, at least as long as $q_{L}>0$, because if this wasn't the case we could increase $w_{H}$ to $w_{H}^{\prime}=w_{H}+\epsilon$, decrease the probability of monitoring to $q_{L}^{\prime}=q_{L}-\alpha(\epsilon)$ where $\alpha(\epsilon)>0$ makes the incentive compatibility bind. If $\alpha(\epsilon)(1-p)\left(\delta W_{L L}-R_{L L}-\delta w_{L}+r_{L}\right)>p \delta \epsilon$ then either decrease $W_{L L}$ or increase $r_{L}$ to make the promise keeping constraint bind. If $\alpha(\epsilon)(1-p)\left(\delta W_{L L}-R_{L L}-\delta w_{L}+r_{L}\right)<p \delta \epsilon$ increase $R_{L L}$ to $R_{L L}^{\prime}=$ $R_{L L}+\beta(\epsilon)$, where $\beta(\epsilon)$ makes the promise keeping constraint bind. Such a new contract would satisfy all other constraints for $\epsilon$ small and expected revenues would change in the first case to $\Pi(w)+\alpha(\epsilon)(1-p) \gamma$ and in the second case to $\Pi(w)+p\left(1+\phi_{H}\right) \epsilon+(1-p) \alpha(\epsilon) \gamma$, where all the other terms vanish because all promised utilities are above $\widehat{w}$. But again, if $w_{H}=\bar{w}$ and $r_{H}=0$ the only possible monitoring probability is $q_{L}=0$.

\section{References}

[1] S.R. Aiyagari and F. Alvarez. Efficient dynamic monitoring of unemployment insurance claims. manuscript, University of Chicago, 1995.

[2] K.C. Border and J. Sobel. Samurai accountant: A theory of auditing and plunder. The Review of economic studies, 54(4):525-540, 1987.

[3] J.H. Boyd, C. Chang, and B.D. Smith. Moral hazard under commercial and universal banking. Journal of Money, Credit \&s Banking, 30(3), 1998.

[4] J.H. Boyd and B.D. Smith. The equilibrium allocation of investment capital in the presence of adverse selection and costly state verification. Economic Theory, 3(3):427-451, 1993.

[5] C. Chang. The dynamic structure of optimal debt contracts. Journal of Economic Theory, 52(1):68-86, 1990.

[6] D. Gale and M. Hellwig. Incentive-compatible debt contracts: The oneperiod problem. The Review of Economic Studies, pages 647-663, 1985.

[7] H. Hopenhayn and I. Werning. Equilibrium default. Manuscript, MIT, 2008. 
[8] T.J. Kehoe and D.K. Levine. Debt-constrained asset markets. The Review of Economic Studies, pages 865-888, 1993.

[9] S. Krasa, T. Sharma, and A.P. Villamil. Bankruptcy and firm finance. Economic Theory, 36(2):239-266, 2008.

[10] C. Monnet and E. Quintin. Optimal contracts in a dynamic costly state verification model. Economic Theory, 26(4):867-885, 2005.

[11] D. Mookherjee and I. Png. Optimal auditing, insurance, and redistribution. The Quarterly Journal of Economics, pages 399-415, 1989.

[12] C. Phelan. Repeated moral hazard and one-sided commitment. Journal of Economic Theory, 66(2):488-506, 1995.

[13] C. Phelan and R.M. Townsend. Computing multi-period, informationconstrained optima. The Review of Economic Studies, pages 853-881, 1991.

[14] L. Popov. Stochastic costly state verification and dynamic contracts. 2007.

[15] B.D. Smith and C. Wang. Repeated insurance relationships in a costly state verification model: With an application to deposit insurance. Journal of Monetary Economics, 42(2):207-240, 1998.

[16] S.E. Spear and C. Wang. When to fire a ceo: optimal termination in dynamic contracts. Journal of Economic Theory, 120(2):239-256, 2005.

[17] J. Thomas and T. Worrall. Self-enforcing wage contracts. The Review of Economic Studies, 55(4):541-553, 1988.

[18] R. Townsend. Optimal contracts and competitive markets with costly state verification. Journal of Economic theory, 21(2):265-93, 1979.

[19] C. Wang. Dynamic costly state verification. Economic Theory, 25(4):887-916, 2005.

[20] D.C. Webb. Two-period financial contracts with private information and costly state verification. The Quarterly Journal of Economics, 107(3):1113-1123, 1992.

[21] S.D. Williamson. Costly monitoring, financial intermediation, and equilibrium credit rationing. Journal of Monetary Economics, 18(2):159$179,1986$. 
[22] S.D. Williamson. Costly monitoring, loan contracts, and equilibrium credit rationing. The Quarterly Journal of Economics, pages 135-145, 1987. 